\title{
On the calculation of energy release rates for cracked laminates with residual stresses
}

\author{
J. A. NAIRN \\ Wood Science and Engineering, Oregon State University, Corvallis, OR 97331, USA \\ E-mail: John.nairn@oregonstate.edu
}

\begin{abstract}
Prior methods for calculating energy release rate in cracked laminates were extended to account for heterogeneous laminates and residual stresses. The method is to partition the crack tip stresses into local bending moments and normal forces. A general equation is then given for the total energy release rate in terms of the crack-tip moments and forces and the temperature difference experienced by the laminate. The analysis method is illustrated by several example test geometries. The examples were verified by comparison to numerical calculations. The residual stress term in the total energy release rate equation was found to be essentially exact in all example calculations.
\end{abstract}

Key words: Residual Stresses, Energy Release Rate, Laminates, Adhesion, Material Point Method

\section{Introduction}

Schapery and Davidson, ${ }^{1}$ Hutchinson and Suo, ${ }^{2}$ and Williams ${ }^{3}$ derived general methods for calculating energy release rates in a variety of laminate specimens from the crack-tip values for bending moments, normal forces, and shear forces. ${ }^{3}$ References [1] and [2] considered heterogeneous beams. Although Ref. [3] considered only homogeneous beams, it was noted that it is trivial to extend it to include any heterogeneous elastic arrangement of the layers. When heterogeneity is present including differential thermal expansion properties and the structure is subjected to a change in temperature, however, there will be residual stresses that are not included in prior analyses and may contribute to energy release rate. This paper extends the prior methods to a general method for calculating the role of residual stresses in fracture of cracked laminates subjected to a uniform change in temperature.

\section{Energy release rate with residual stresses}

Figure 1 shows a general laminated or multilayered structure having a crack with moments $\left(M_{1}^{(0)}, M_{2}^{(0)}\right.$, and $\left.M_{3}^{(0)}\right)$ applied to each arm and axial forces $\left(N_{1}, N_{2}\right.$, and $\left.N_{3}\right)$ on each arm resolved into point loads applied at arbitrary locations $\left(n_{1}, n_{2}\right.$, and $\left.n_{3}\right)$. By force and moment balance, the resultants local to the small crack tip region of length $\delta L$ are:

$$
\begin{aligned}
& N_{3}=N_{1}+N_{2} \\
& M_{1}=M_{1}^{(0)}+N_{1}\left(\frac{h_{1}}{2}-n_{1}\right) \\
& M_{2}=M_{2}^{(0)}+N_{2}\left(\frac{h_{2}}{2}-n_{2}\right) \\
& M_{3}=M_{1}+M_{2}-N_{1}\left(h_{2}-n_{3}+n_{1}\right)+N_{2}\left(n_{3}-n_{2}\right)
\end{aligned}
$$

The "layers" indicate the possibility of multiple layers within each arm of the general composite beam. Shear forces may be included (as discussed in Ref. [3]), but are not included here because prior results on laminate residual stress effects suggest that shear corrections are not needed to analyze residual stress energy 


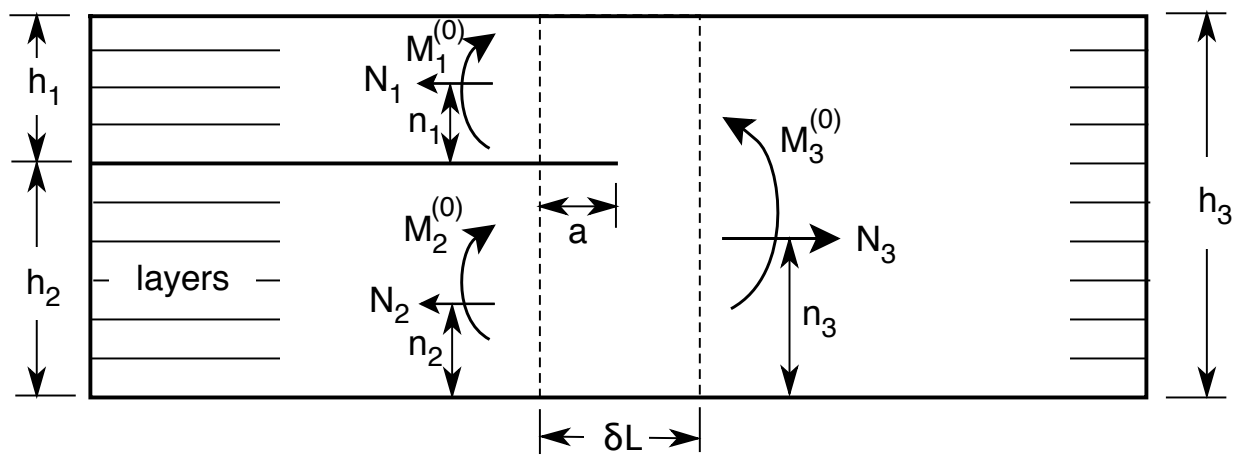

Fig. 1. A section of a general laminate or multilayered structure with a crack. The laminate is divided into three multilayered arms with total thicknesses $h_{1}, h_{2}$, and $h_{3}$. Arms 1 and 2 are above and below the crack; arm 3 is the intact portion of the laminate. In the crack-tip region, the arms have applied moments, $M_{1}^{(0)}, M_{2}^{(0)}$, and $M_{3}^{(0)}$, and normal forces. $N_{1}, N_{2}$, and $N_{3}$. The normal forces are applied at positions $n_{1}, n_{2}$, and $n_{3}$, above the bottoms of the arms.

release rates. ${ }^{4}$ Each sublaminate (cracked arms 1 and 2 or intact beam 3 ) is treated as a beam having having curvature and axial strain given by

$$
\begin{aligned}
\kappa & =C_{\kappa}^{(i)} M+D^{(i)} N+\alpha_{\kappa}^{(i)} \Delta T \\
\varepsilon_{0} & =D^{(i)} M+C_{\varepsilon}^{(i)} N+\alpha_{\varepsilon}^{(i)} \Delta T
\end{aligned}
$$

where $C_{\kappa}^{(i)}$ and $C_{\varepsilon}^{(i)}$ are compliances for curvature, $\kappa$, and net axial strain at the midplane of each laminate, $\varepsilon_{0}, D^{(i)}$ is the coupling compliance between strains and curvature, and $\alpha_{\kappa}^{(i)}$ and $\alpha_{\varepsilon}^{(i)}$ are curvature and linear thermal expansion coefficients of each laminate. $\Delta T$ is the uniform temperature differential leading to residual stresses. The moments in Fig. 1 are positive and induce positive $\kappa$ defined here as upward curvature. The coupling term, $D^{(i)}$, and the curvature thermal expansion, $\alpha_{\kappa}^{(i)}$, are zero whenever an arm is either homogeneous or a symmetric laminate. They are non-zero only for non-symmetric, heterogeneous laminate arms.

The exact and general energy release rate for this composite of thickness $B$ with residual stresses subjected only to traction loads, $\vec{T}^{0}$, is given by: ${ }^{5,6}$

$$
G=\frac{1}{B} \frac{d}{d a}\left(\frac{1}{2} \int_{S} \vec{T}^{0} \cdot \vec{u}^{m} d S+\int_{S} \vec{T}^{0} \cdot \vec{u}^{r} d S+\frac{1}{2} \int_{V} \boldsymbol{\sigma}^{r} \cdot \boldsymbol{\alpha} \Delta T d V\right)
$$

where $\vec{u}^{m}$ and $\vec{u}^{r}$ are displacements due to mechanical and residual stresses and $\boldsymbol{\sigma}^{r}$ are the residual stresses. The first term is the strain energy due to mechanical loads and thus identical to prior analyses. ${ }^{1-3}$ Including heterogeneous sublaminates, considering the small zone of length $\delta L$ around the crack tip, and superposing energy due to moments and normal forces, the first term is

$$
\begin{array}{r}
\frac{1}{2} \int_{S} \vec{T}^{0} \cdot \vec{u}^{m} d S=\frac{1}{2} C_{\kappa}^{(1)} M_{1}^{2} a+\frac{1}{2} C_{\kappa}^{(2)} M_{2}^{2} a+\frac{1}{2} C_{\kappa}^{(3)} M_{3}^{2}(\delta L-a)+D^{(1)} M_{1} N_{1} a+D^{(2)} M_{2} N_{2} a \\
+D^{(3)} M_{3} N_{3} a+\frac{1}{2} C_{\varepsilon}^{(1)} N_{1}^{2} a+\frac{1}{2} C_{\varepsilon}^{(2)} N_{2}^{2} a+\frac{1}{2} C_{\varepsilon}^{(3)} N_{3}^{2}(\delta L-a)
\end{array}
$$

The $M_{i}^{2}$ and $N_{i}^{2}$ terms are analogous to mechanical terms in Refs. [1-3]. The cross-terms arise due to straincurvature coupling. These coupling terms are zero for homogeneous or symmetric sublaminates, but they are non-zero if the arms are non-symmetric and heterogeneous. The coupling terms are included in Ref. [1], but they were omitted in Ref. [2]. They also were omitted in Ref. [3] because that analysis was limited to homogeneous beams where the coupling terms are zero.

The second term in Eq. (7) is virtual work between the mechanical tractions and the residual displacements:

$$
\int_{S} \vec{T}^{0} \cdot \vec{u}^{r} d S=M_{1} \alpha_{\kappa}^{(1)} \Delta T a+M_{2} \alpha_{\kappa}^{(2)} \Delta T a+M_{3} \alpha_{\kappa}^{(3)} \Delta T(\delta L-a)+
$$




$$
N_{1} \alpha_{\varepsilon}^{(1)} \Delta T a+N_{2} \alpha_{\varepsilon}^{(2)} \Delta T a+N_{3} \alpha_{\varepsilon}^{(3)} \Delta T(\delta L-a)
$$

For a single orthotropic sublaminate spanning $x$ from $x_{0}$ to $x_{1}$, the last term in Eq. (7) simplifies to

$$
\frac{1}{2} \int_{V} \boldsymbol{\sigma}^{r} \cdot \boldsymbol{\alpha} \Delta T d V=\int_{x_{0}}^{x_{1}}\left(B \int_{-h / 2}^{h / 2} \sigma_{x x}^{r} \alpha(y) \Delta T d y\right) d x
$$

where $\sigma_{x x}^{r}$ is residual stress in the $x$ direction and $\alpha(y)$ is the position-dependent thermal expansion coefficient. The term involving $\sigma_{y y}^{r}$ is zero after integration over $x$ because of zero force in the $y$ direction and the layered structure of the material. As derived in Appendix A, the result for the third term can be written as

$$
\begin{array}{r}
\frac{1}{2} \int_{V} \boldsymbol{\sigma}^{r} \cdot \boldsymbol{\alpha} \Delta T d V=\frac{a \Delta T^{2}}{2}\left(-B h_{1} E_{0}^{(1)} \sigma_{\alpha E}^{(1)^{2}}+\frac{\alpha_{\kappa}^{(1)^{2}}}{C_{\kappa}^{(1)}}\right)+\frac{a \Delta T^{2}}{2}\left(-B h_{2} E_{0}^{(2)}{\sigma_{\alpha E}^{(2)^{2}}}^{2} \frac{\alpha_{\kappa}^{(2)^{2}}}{C_{\kappa}^{(2)}}\right) \\
+\frac{(\delta L-a) \Delta T^{2}}{2}\left(-B h_{3} E_{0}^{(3)} \sigma_{\alpha E}^{(3)^{2}}+\frac{\alpha_{\kappa}^{(3)^{2}}}{C_{\kappa}^{(3)}}\right)
\end{array}
$$

where $E_{0}^{(i)}$ is the rule-of-mixtures modulus in sublaminate $\mathrm{i}$ and ${\sigma_{\alpha E}^{(i)}}^{2}$ is the variance of the modulus-weighted thermal expansion coefficient in sublaminate $\mathrm{i}$ (see definitions in Appendix A).

Subsituting all terms into Eq. (7) and differentiating gives the general result for energy release rate in a cracked laminate with residual stresses:

$$
\begin{aligned}
G=\frac{1}{2 B}( & C_{\kappa}^{(1)} M_{1}^{2}+C_{\kappa}^{(2)} M_{2}^{2}-C_{\kappa}^{(3)} M_{3}^{2}+C_{\varepsilon}^{(1)} N_{1}^{2}+C_{\varepsilon}^{(2)} N_{2}^{2}-C_{\varepsilon}^{(3)} N_{3}^{2}+2 D^{(1)} M_{1} N_{1}+2 D^{(2)} M_{2} N_{2} \\
& \left.-2 D^{(3)} M_{3} N_{3}\right)+\frac{\Delta T}{B}\left(\alpha_{\kappa}^{(1)} M_{1}+\alpha_{\kappa}^{(2)} M_{2}-\alpha_{\kappa}^{(3)} M_{3}+\alpha_{\varepsilon}^{(1)} N_{1}+\alpha_{\varepsilon}^{(2)} N_{2}-\alpha_{\varepsilon}^{(3)} N_{3}\right) \\
& +\frac{\Delta T^{2}}{2 B}\left(\frac{\alpha_{\kappa}^{(1)^{2}}}{C_{\kappa}^{(1)}}-B h_{1} E_{0}^{(1)}{\sigma_{\alpha E}^{(1)}}^{2}+\frac{\alpha_{\kappa}^{(2)}}{C_{\kappa}^{(2)}}-B h_{2} E_{0}^{(2)}{\sigma_{\alpha E}^{(2)^{2}}}^{2}-\frac{\alpha_{\kappa}^{(3)^{2}}}{C_{\kappa}^{(3)}}+B h_{3} E_{0}^{(3)} \sigma_{\alpha E}^{(3)^{2}}\right)
\end{aligned}
$$

When residual stresses are ignored, $\Delta T=0$, this result reduces to prior results . For homogeneous beams with modulus $E$ and thermal expansion coefficient $\alpha\left(C_{\kappa}^{(i)}=1 /\left(E I^{(i)}\right), C_{\varepsilon}^{(i)}=1 /\left(E A^{(i)}\right), \alpha_{\varepsilon}^{(i)}=\alpha\right.$, and $D^{(i)}=\alpha_{\kappa}^{(i)}=\sigma_{\alpha E}^{(i)^{2}}=0$ ), this result reduces to the results in Refs. [2] and [3] for total energy release rate. For heterogeneous beams, this result reduces to the results in Ref. [1] for total energy release rate. Equation (12) thus extends all prior results for residual stresses, which is the purpose of this paper.

\section{Mode partitioning}

Willliams ${ }^{3}$ partitioned applied moments into mode I and mode II moments, $M_{I}$ and $M_{I I}$, by considered the mode II part to be the moments required to get equal curvature in the two arms and the remaining moments to be mode I. The energy release rate was then partitioned into $G=G_{I}+G_{I I}$ where $G_{I}$ was due to $M_{I}, G_{I I}$ was due to $M_{I I}$, and there was no coupling between $M_{I}$ and $M_{I I}$. The same methods did not work here. First, in the absence of applied loads, there are no moments and forces to partition and the temperature is always uniform over the laminate. Second, the partitioning in Ref. [3] is not unique. There are an infinite number of ways to partition applied moments into $M_{I}$ and $M_{I I}$ such that $G$ partitions into decoupled $G_{I}$ and $G_{I I}$. Furthermore, the choice of equal curvature for the mode II component was found to disagree with finite element results. Schapery has pointed out that there is not enough information in beam analyses or plate analyses to decompose total energy release rate. There is an undetermined constant that has to be determined by other means. For example, Hutchinson and $\mathrm{Suo}^{2}$ used numerical calculations to calibrate mode decomposition equations. Because no partitioning based on beam analyses is available, the results in this paper focused on total energy release rate. The only comments in this paper about mode partitioning are in reference to numerical calculations. 

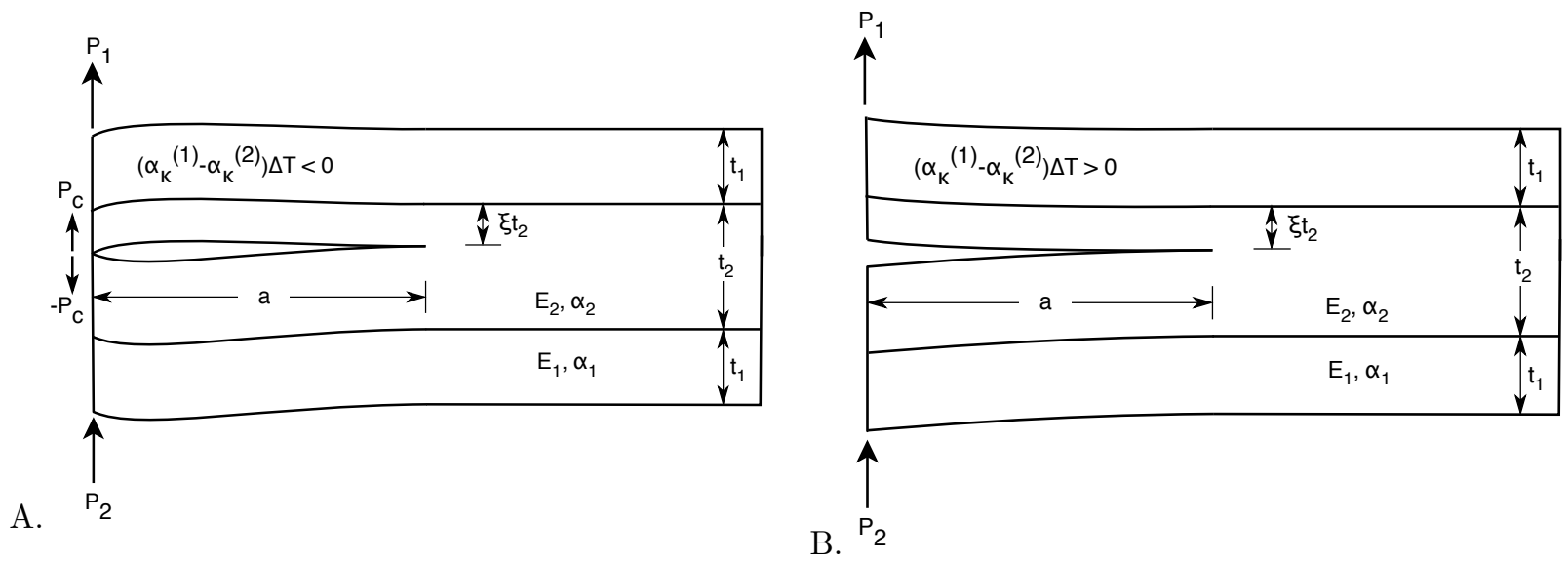

Fig. 2. A symmetric three-layered specimen with a crack at an arbitrary location in the middle layer. The surface layers have modulus, thermal expansion coefficient, and thickness of $E_{1}, \alpha_{1}$, and $t_{1}$; the middle layer has properties $E_{2}, \alpha_{2}$, and $t_{2}$. The ends of the arms are loaded with loads $P_{1}$ and $P_{2}$. A. When residual stresses cause the cracked arms to curve toward each other, there may be a contact load $P_{c}$ at the ends of the arms. B. When residual stresses cause the cracked arms to separate, there will be no contact.

\section{Examples}

\subsection{END-LOADED ADHESIVE SPECIMEN}

Figure 2 shows a symmetric adhesive specimen with arbitrary loads on the two arms, a crack located at arbitrary position $\xi t_{2}$ from the top interface $(0 \leq \xi \leq 1)$, and crack propagation in a self-similar manner. Some key terms are

$$
\begin{gathered}
\alpha_{\kappa}^{(3)}=0, \quad N_{i}=0, \quad \lambda_{1}=\frac{\lambda}{\xi}, \quad \lambda_{2}=\frac{\lambda}{1-\xi} \\
h_{1} E_{0}^{(1)}{\sigma_{\alpha E}^{(1)}}^{2}=\frac{E_{1} t_{1} \xi \Delta \alpha^{2}}{\xi+R \lambda}, \quad h_{2} E_{0}^{(2)}{\sigma_{\alpha E}^{(2)}}^{2}=\frac{E_{1} t_{1}(1-\xi) \Delta \alpha^{2}}{1-\xi+R \lambda}, \quad h_{3} E_{0}^{(3)}{\sigma_{\alpha E}^{(3)}}^{2}=\frac{2 E_{1} t_{1} \Delta \alpha^{2}}{1+2 R \lambda}
\end{gathered}
$$

where $\lambda=t_{1} / t_{2}$ and $R=E_{1} / E_{2}$ (see Appendix A for common calculations needed for two- and three-layer arms and for the definition of $\lambda_{i}$ ).

The actual end loads, and therefore crack-tip moments, depend on whether or not there is contact between the two arms. The applied forces have to be adjusted to account for contact forces before calculating crack-tip moments. By integrating the arm curvature equations $\left(\kappa=d^{2} v(x) / d x^{2}=C_{\kappa}^{(i)} P_{i} x+\alpha_{\kappa}^{(i)} \Delta T\right)$ and subtracting, the crack-opening-displacement can be derived to be

$$
\delta(\zeta)=\frac{a^{2}}{6}(1-\zeta)^{2}\left[a\left(C_{\kappa}^{(1)} P_{1}-C_{\kappa}^{(2)} P_{2}\right)(2+\zeta)+\left(\alpha_{\kappa}^{(1)}-\alpha_{\kappa}^{(2)}\right) \Delta T\right]
$$

where $\zeta=x / a$ and $x=0$ is at the ends of the arms while $x=a$ is at the crack tip. If $\left(\alpha_{\kappa}^{(1)}-\alpha_{\kappa}^{(2)}\right) \Delta T<0$ (e.g., thermal expansion coefficient on the adhesive is higher than the adherend and the sample is cooled), the two arms will bend toward each other due to residual stresses (see Fig. 2A). There will be either contact at the ends or the loads will be sufficient to separate the two arms. From $\delta(\zeta)$, the arms will separate $(\delta(\zeta)>0$ for $0 \leq \zeta<1)$ when

$$
C_{\kappa}^{(1)} P_{1}-C_{\kappa}^{(2)} P_{2}>-\frac{3\left(\alpha_{\kappa}^{(1)}-\alpha_{\kappa}^{(2)}\right) \Delta T}{2 a}
$$

resulting in zero contact force and

$$
M_{1}=P_{1} a, \quad M_{2}=P_{2} a, \quad M_{3}=\left(P_{1}+P_{2}\right) a
$$


The energy release rate will be $G=G_{a}$ where

$$
\begin{aligned}
G_{a}=\frac{1}{2 B} & \left(P_{1} a \sqrt{C_{\kappa}^{(1)}}+\frac{\alpha_{\kappa}^{(1)} \Delta T}{\sqrt{C_{\kappa}^{(1)}}}\right)^{2}+\frac{1}{2 B}\left(P_{2} a \sqrt{C_{\kappa}^{(2)}}+\frac{\alpha_{\kappa}^{(2)} \Delta T}{\sqrt{C_{\kappa}^{(2)}}}\right)^{2} \\
& -\frac{1}{2 B}\left(P_{1}+P_{2}\right)^{2} a^{2} C_{\kappa}^{(3)}+\frac{E_{1} t_{1} R \lambda(1-2 \xi)^{2} \Delta \alpha^{2} \Delta T^{2}}{2(1+2 R \lambda)(\xi+R \lambda)(1-\xi+R \lambda)}
\end{aligned}
$$

In contrast, when

$$
C_{\kappa}^{(1)} P_{1}-C_{\kappa}^{(2)} P_{2}=-\frac{3\left(\alpha_{\kappa}^{(1)}-\alpha_{\kappa}^{(2)}\right) \Delta T}{2 a}
$$

the ends of the arms will just be in contact at $\zeta=0$ with zero contact force between the arms; $\delta(\zeta)$ will remain positive over the remainder of the crack surface. If $C_{\kappa}^{(1)} P_{1}-C_{\kappa}^{(2)} P_{2}$ decreases, a contact force, $P_{c}$, will develop and the net forces in the two arms will be $P_{1}+P_{c}$ and $P_{2}-P_{c}$ (see Fig. 2A). The contact force can be determined from the requirement that $\delta(0)$ due to net forces remains zero or

$$
C_{\kappa}^{(1)}\left(P_{1}+P_{c}\right)-C_{\kappa}^{(2)}\left(P_{2}-P_{c}\right)=-\frac{3\left(\alpha_{\kappa}^{(1)}-\alpha_{\kappa}^{(2)}\right) \Delta T}{2 a}
$$

which leads to

$$
P_{c}=-\frac{3\left(\alpha_{\kappa}^{(1)}-\alpha_{\kappa}^{(2)}\right) \Delta T}{2 a\left(C_{\kappa}^{(1)}+C_{\kappa}^{(2)}\right)}-\frac{C_{\kappa}^{(1)} P_{1}-C_{\kappa}^{(2)} P_{2}}{C_{\kappa}^{(1)}+C_{\kappa}^{(2)}}
$$

The net crack-tip moments become

$$
M_{1}=\left(P_{1}+P_{c}\right) a, \quad M_{2}=\left(P_{2}-P_{c}\right) a, \quad M_{3}=\left(P_{1}+P_{2}\right) a
$$

The energy release rate is $G=G_{b}$ where

$$
\begin{aligned}
G_{b}=\frac{\left(P_{1}+P_{2}\right)^{2} a^{2}}{2 B}\left(\frac{C_{\kappa}^{(1)} C_{\kappa}^{(2)}}{C_{\kappa}^{(1)}+C_{\kappa}^{(2)}}-C_{\kappa}^{(3)}\right)+\frac{\left(P_{1}+P_{2}\right) a}{B} \frac{\left(\alpha_{\kappa}^{(1)} C_{\kappa}^{(1)}+\alpha_{\kappa}^{(2)} C_{\kappa}^{(2)}\right) \Delta T}{C_{\kappa}^{(1)}+C_{\kappa}^{(2)}} \\
+\frac{\Delta T^{2}}{2 B}\left[\frac{\alpha_{\kappa}^{(1)}}{C_{\kappa}^{(1)}}+\frac{\alpha_{\kappa}^{(2)}}{C_{\kappa}^{(2)}}-\frac{3\left(\alpha_{\kappa}^{(1)}-\alpha_{\kappa}^{(2)}\right)^{2}}{4\left(C_{\kappa}^{(1)}+C_{\kappa}^{(2)}\right)}+\frac{B E_{1} t_{1} R \lambda(1-2 \xi)^{2} \Delta \alpha^{2}}{(1+2 R \lambda)(\xi+R \lambda)(1-\xi+R \lambda)}\right]
\end{aligned}
$$

If $\left(\alpha_{\kappa}^{(1)}-\alpha_{\kappa}^{(2)}\right) \Delta T>0$ (e.g., thermal expansion coefficient on the adhesive is higher than the adherend and the sample is heated), the two arms will separate due to residual stresses (see Fig. 2B). There will either be no contact or there will be surface overlap in the region near the crack tip. If

$$
C_{\kappa}^{(1)} P_{1}-C_{\kappa}^{(2)} P_{2} \geq-\frac{\left(\alpha_{\kappa}^{(1)}-\alpha_{\kappa}^{(2)}\right) \Delta T}{a}
$$

it can be shown that the arms separate $(\delta(\zeta)>0$ for $0 \leq \zeta<1)$. The net forces are $P_{1}$ and $P_{2}$ leading to the energy release rate in Eq. (18). In contrast, if

$$
C_{\kappa}^{(1)} P_{1}-C_{\kappa}^{(2)} P_{2}<-\frac{\left(\alpha_{\kappa}^{(1)}-\alpha_{\kappa}^{(2)}\right) \Delta T}{a}
$$

there will be a region near the crack tip where $\delta(\zeta)<0$. This contact will induce an indeterminate contact force region that changes in length as the load changes. The equations in this paper do not account for these contact stress and therefore this regime was not analyzed.

Finally, the thermal energy release rate, or the energy release rate in the absence of mechanical loads $\left(P_{1}=P_{2}=0\right)$, is

$$
G_{a}=\frac{\Delta T^{2}}{2 B}\left[\frac{\alpha_{\kappa}^{(1)^{2}}}{C_{\kappa}^{(1)}}+\frac{\alpha_{\kappa}^{(2)^{2}}}{C_{\kappa}^{(2)}}+\frac{B E_{1} t_{1} R \lambda(1-2 \xi)^{2} \Delta \alpha^{2}}{(1+2 R \lambda)(\xi+R \lambda)(1-\xi+R \lambda)}\right]
$$


when the arms separate (or when $\left(\alpha_{\kappa}^{(1)}-\alpha_{\kappa}^{(2)}\right) \Delta T>0$ ), but

$$
G_{b}=\frac{\Delta T^{2}}{2 B}\left[\frac{\alpha_{\kappa}^{(1)^{2}}}{C_{\kappa}^{(1)}}+\frac{\alpha_{\kappa}^{(2)^{2}}}{C_{\kappa}^{(2)}}-\frac{3\left(\alpha_{\kappa}^{(1)}-\alpha_{\kappa}^{(2)}\right)^{2}}{4\left(C_{\kappa}^{(1)}+C_{\kappa}^{(2)}\right)}+\frac{B E_{1} t_{1} R \lambda(1-2 \xi)^{2} \Delta \alpha^{2}}{(1+2 R \lambda)(\xi+R \lambda)(1-\xi+R \lambda)}\right]
$$

when the arms are in contact (or when $\left(\alpha_{\kappa}^{(1)}-\alpha_{\kappa}^{(2)}\right) \Delta T<0$ ). The thermal energy release rates are independent of crack length.

\subsection{Mode I AdHesive SPECIMEN}

A mode I style test applies equal but opposite loads to the arms ends or $P_{1}=-P_{2}=P$. This special case can be written down from the general results in the previous section. If $\left(\alpha_{\kappa}^{(1)}-\alpha_{\kappa}^{(2)}\right) \Delta T<0$ and $P \geq P^{*}$ or $\left(\alpha_{\kappa}^{(1)}-\alpha_{\kappa}^{(2)}\right) \Delta T>0$ and $P \geq 2 P^{*} / 3$ then the arms will not be in contact and

$$
G_{a}=\frac{1}{2 B}\left(P a \sqrt{C_{\kappa}^{(1)}}+\frac{\alpha_{\kappa}^{(1)} \Delta T}{\sqrt{C_{\kappa}^{(1)}}}\right)^{2}+\frac{1}{2 B}\left(P a \sqrt{C_{\kappa}^{(2)}}-\frac{\alpha_{\kappa}^{(2)} \Delta T}{\sqrt{C_{\kappa}^{(2)}}}\right)^{2}+\frac{E_{1} t_{1} R \lambda(1-2 \xi)^{2} \Delta \alpha^{2} \Delta T^{2}}{2(1+2 R \lambda)(\xi+R \lambda)(1-\xi+R \lambda)}
$$

If $\left(\alpha_{\kappa}^{(1)}-\alpha_{\kappa}^{(2)}\right) \Delta T<0$ and $P<P^{*}$ then the arms will contact at the arm ends and

$$
G_{b}=\frac{\Delta T^{2}}{2 B}\left[\frac{\alpha_{\kappa}^{(1)^{2}}}{C_{\kappa}^{(1)}}+\frac{\alpha_{\kappa}^{(2)^{2}}}{C_{\kappa}^{(2)}}-\frac{3\left(\alpha_{\kappa}^{(1)}-\alpha_{\kappa}^{(2)}\right)^{2}}{4\left(C_{\kappa}^{(1)}+C_{\kappa}^{(2)}\right)}+\frac{B E_{1} t_{1} R \lambda(1-2 \xi)^{2} \Delta \alpha^{2}}{(1+2 R \lambda)(\xi+R \lambda)(1-\xi+R \lambda)}\right]
$$

If $\left(\alpha_{\kappa}^{(1)}-\alpha_{\kappa}^{(2)}\right) \Delta T>0$ and $P<2 P^{*} / 3$ then this analysis does not apply. In these equations

$$
P^{*}=-\frac{3\left(\alpha_{\kappa}^{(1)}-\alpha_{\kappa}^{(2)}\right) \Delta T}{2 a\left(C_{\kappa}^{(1)}+C_{\kappa}^{(2)}\right)}
$$

Note that $G_{b}$ is independent of applied load. Thus for $\left(\alpha_{\kappa}^{(1)}-\alpha_{\kappa}^{(2)}\right) \Delta T<0, G$ will be constant for $P<P^{*}$ but will increase by Eq. (28) when $P \geq P^{*}$. Also note that $P^{*}$ is positive when $\left(\alpha_{\kappa}^{(1)}-\alpha_{\kappa}^{(2)}\right) \Delta T<0$, but negative when $\left(\alpha_{\kappa}^{(1)}-\alpha_{\kappa}^{(2)}\right) \Delta T>0$. Thus, the only regime where the analysis does not apply is for negative loads $\left(P<2 P^{*} / 3<0\right.$ because $\left.\left(\alpha_{\kappa}^{(1)}-\alpha_{\kappa}^{(2)}\right) \Delta T>0\right)$, which is not a loading region ever explored during end-loading experiments on adhesive specimens.

For a crack at the midplane, $\xi=1 / 2, C_{\kappa}^{(2)}=C_{\kappa}^{(1)}$, and $\alpha_{\kappa}^{(2)}=-\alpha_{\kappa}^{(1)}$; the energy release rates reduce to

$$
G_{a}=\frac{1}{B}\left(P a \sqrt{C_{\kappa}^{(1)}}+\frac{\alpha_{\kappa}^{(1)} \Delta T}{\sqrt{C_{\kappa}^{(1)}}}\right)^{2}, \quad G_{b}=\frac{\alpha_{\kappa}^{(1)^{2}} \Delta T^{2}}{4 B C_{\kappa}^{(1)}}, \quad \text { and } \quad P^{*}=-\frac{3 \alpha_{\kappa}^{(1)} \Delta T}{2 a C_{\kappa}^{(1)}}
$$

The result for $G_{a}$ agrees with the results in Ref. [4]. The result for $G_{b}$ extends the prior result to be correct for loads less than $P^{*}$. The loading is pure mode I when $\xi=1 / 2$, but otherwise the asymmetry will result in mixed-mode loading.

For a crack at the lower interface, $\xi=1$ and $\alpha_{\kappa}^{(2)}=0$; the energy release rates reduce to

$$
\begin{aligned}
G_{a} & =\frac{1}{2 B}\left(P a \sqrt{C_{\kappa}^{(1)}}+\frac{\alpha_{\kappa}^{(1)} \Delta T}{\sqrt{C_{\kappa}^{(1)}}}\right)^{2}+\frac{P^{2} a^{2} C_{\kappa}^{(2)}}{2 B}+\frac{E_{1} t_{1} \Delta \alpha^{2} \Delta T^{2}}{2(1+2 R \lambda)(1+R \lambda)} \\
G_{b} & =\frac{\Delta T^{2}}{2}\left(\frac{\alpha_{\kappa}^{(1)}}{B C_{\kappa}^{(1)}}+\frac{E_{1} t_{1} \Delta \alpha^{2}}{(1+2 R \lambda)(1+R \lambda)}-\frac{3 \alpha_{\kappa}^{(1)}}{4 B\left(C_{\kappa}^{(1)}+C_{\kappa}^{(2)}\right)}\right) \\
P^{*} & =-\frac{3 \alpha_{\kappa}^{(1)} \Delta T}{2 a\left(C_{\kappa}^{(1)}+C_{\kappa}^{(2)}\right)}
\end{aligned}
$$


These results are identical to the results in Ref. [7]. The general results are symmetric about $\xi$; thus the results at the upper interface are the same, but are calculated by exchanging superscripts (1) for (2).

\subsection{MOdE II ADHESIVE SPECIMEN}

A mode II style test applies a load to one arm and relies on contact to transmit the load to the second arm; thus $P_{1}=0$ and $P_{2}=P$. This special case can be written down from the general results given above. If $\left(\alpha_{\kappa}^{(1)}-\alpha_{\kappa}^{(2)}\right) \Delta T<0$ and $P<P^{*}$ or $\left(\alpha_{\kappa}^{(1)}-\alpha_{\kappa}^{(2)}\right) \Delta T>0$ and $P \leq 2 P^{*} / 3$ then the arms will not be in contact and

$$
G_{a}=\frac{\alpha_{\kappa}^{(1)^{2}} \Delta T^{2}}{2 B C_{\kappa}^{(1)}}+\frac{1}{2 B}\left(P a \sqrt{C_{\kappa}^{(2)}}+\frac{\alpha_{\kappa}^{(2)} \Delta T}{\sqrt{C_{\kappa}^{(2)}}}\right)^{2}-\frac{P^{2} a^{2} C_{\kappa}^{(3)}}{2 B}+\frac{E_{1} t_{1} R \lambda(1-2 \xi)^{2} \Delta \alpha^{2} \Delta T^{2}}{2(1+2 R \lambda)(\xi+R \lambda)(1-\xi+R \lambda)}
$$

If $\left(\alpha_{\kappa}^{(1)}-\alpha_{\kappa}^{(2)}\right) \Delta T<0$ and $P \geq P^{*}$ then the arms will contact at the arm ends and

$$
\begin{aligned}
G_{b}=\frac{P^{2} a^{2}}{2 B} & \left(\frac{C_{\kappa}^{(1)} C_{\kappa}^{(2)}}{C_{\kappa}^{(1)}+C_{\kappa}^{(2)}}-C_{\kappa}^{(3)}\right)+\frac{P a}{B} \frac{\left(\alpha_{\kappa}^{(1)} C_{\kappa}^{(1)}+\alpha_{\kappa}^{(2)} C_{\kappa}^{(2)}\right) \Delta T}{C_{\kappa}^{(1)}+C_{\kappa}^{(2)}} \\
& +\frac{\Delta T^{2}}{2 B}\left[\frac{\alpha_{\kappa}^{(1)^{2}}}{C_{\kappa}^{(1)}}+\frac{\alpha_{\kappa}^{(2)}}{C_{\kappa}^{(2)}}-\frac{3\left(\alpha_{\kappa}^{(1)}-\alpha_{\kappa}^{(2)}\right)^{2}}{\left(C_{\kappa}^{(1)}+C_{\kappa}^{(2)}\right)}+\frac{B E_{1} t_{1} R \lambda(1-2 \xi)^{2} \Delta \alpha^{2}}{(1+2 R \lambda)(\xi+R \lambda)(1-\xi+R \lambda)}\right]
\end{aligned}
$$

If $\left(\alpha_{\kappa}^{(1)}-\alpha_{\kappa}^{(2)}\right) \Delta T>0$ and $P>2 P^{*} / 3$ then this analysis does not apply. In these equations

$$
P^{*}=\frac{3\left(\alpha_{\kappa}^{(1)}-\alpha_{\kappa}^{(2)}\right) \Delta T}{2 a C_{\kappa}^{(2)}}
$$

In the absence of mechanical loads $(P=0)$, the thermal energy release rate in the mode II style test is identical to the mode I style test, as expected.

For a crack at the midplane, $\xi=1 / 2, C_{\kappa}^{(2)}=C_{\kappa}^{(1)}$, and $\alpha_{\kappa}^{(2)}=-\alpha_{\kappa}^{(1)}$; the energy release rates reduce to

$$
\begin{aligned}
G_{a} & =\frac{P^{2} a^{2}}{2 B}\left(C_{\kappa}^{(1)}-C_{\kappa}^{(3)}\right)+\frac{P a \alpha_{\kappa}^{(1)} \Delta T}{B}+\frac{\alpha_{\kappa}^{(1)^{2}} \Delta T^{2}}{B C_{\kappa}^{(1)}} \\
G_{b} & =\frac{P^{2} a^{2}}{4 B}\left(C_{\kappa}^{(1)}-2 C_{\kappa}^{(3)}\right)+\frac{\alpha_{\kappa}^{(1)^{2}} \Delta T^{2}}{4 B C_{\kappa}^{(2)}} \\
P^{*} & =\frac{3 \alpha_{\kappa}^{(1)} \Delta T}{a C_{\kappa}^{(1)}}
\end{aligned}
$$

For the conditions that lead to $G_{a}$, there will be no contact between the arms, which thwarts the intent of the test to load both arms. These loading conditions will differ significantly from pure mode II. The conditions that lead to $G_{b}$ will result in a contact force of

$$
P_{c}=\frac{P}{2}-\frac{3 \alpha_{\kappa}^{(1)} \Delta T}{2 a C_{\kappa}^{(1)}}
$$

In the absence of residual stresses, the loads will partition into $P / 2$ on each arm and the test will be pure mode II. When residual stresses are present, they will alter the load partitioning causing the specimen to deviate from pure mode II loading. Residual stresses add equal but opposite contact stresses thereby adding a mode I component.

For homogeneous beams, these results reduce to the mode II energy release rate ${ }^{3}$ of

$$
G=\frac{9}{4} \frac{P^{2} a^{2}}{B^{2} E h^{3}}
$$


where $2 h=2 t_{1}+t_{2}$. This result applies with or without residual stresses. In other words, $\Delta T \neq 0$ has no affect on energy release rate for a homogeneous beam.

For $\xi \neq 1 / 2$, the results for the mode II style test are no longer symmetric about $\xi=1 / 2$. Thus the energy release rate for a crack at the top interface $(\xi=0)$ will differ from the energy release rate for a crack at the bottom interface $(\xi=1)$. The results for any value of $\xi$ are easily generated from the general results and are not repeated here.

\subsection{Mode II AdHeSIVE SPECIMEN With FRICTION}

In end-loaded flexure tests for mode II fracture $\left(P_{1}=0\right.$ and $\left.P_{2}=P\right)$ of homogeneous beams, the load will partition into $P / 2$ on each arm, the curvature of the two arms will be identical, and there will be contact along the entire crack surface. This contact raises the concern that frictional effects may influence the results. ${ }^{8}$ When the beams are heterogeneous and there are residual stresses, most experimental conditions will still have contact, but the contact will only be at the loading point (see Fig. 3). There will still be friction, but analysis of point-contact friction is more straightforward than analysis of contact along the entire crack surface. This section presents an analysis for mode II loading in the presence of residual stresses and friction. By taking the limit as residual stresses go to zero, an equation can be derived for the case of contact along the entire crack surface. The analysis is given for a midplane crack $(\xi=1 / 2)$, but the approach could easily be extended to any crack location.

The arm forces and moments for mode II loading with friction are shown in Fig. 3. A load $P$ is applied to arm 2. Load is transferred to arm 1 by a contact load $P_{c}$. In mode II loading, the arms will slide relative to each other inducing a frictional load that can be modeled as equal, but opposite, normal loads on arms 1 and 2 applied at the contact point. The loading conditions are thus given by

$$
N_{1}=-\mu P_{c} \quad n_{1}=0 \quad N_{2}=\mu P_{c} \quad n_{2}=h \quad N_{3}=0
$$

These loads, and therefore this analysis, assume frictional slip does occur. If there is no slip, $N_{1}$ and $N_{2}$ will be smaller and the effect on energy release rate will be smaller. The resulting moments on the arms are

$$
M_{1}=P_{c}-\frac{\mu h P_{c}}{2} \quad M_{2}=\left(P-P_{c}\right) a-\frac{\mu h P_{c}}{2} \quad M_{3}=P a-\mu h P_{c}
$$

The contact load $P_{c}$ is found by requiring zero crack-opening displacement at the load point. Because the forces and moments induced by friction affect the two arms equally, the contact analysis in the general

adhesive specimen is still valid. For the midplane crack, $P_{c}$ is thus given in Eq. (41). Substituting into Eq. (12), expanding the result in terms of a dimensionless frictional term $\mu h /(2 a)$, and using $C_{\varepsilon}^{(2)}=C_{\varepsilon}^{(1)}$ and $D^{(2)}=-D^{(1)}$ for a midplane crack, the straightforward, albeit tedious, process soon gives

$$
\begin{aligned}
G_{b}=G_{b}^{0}- & \frac{P a^{2}}{B}\left(\frac{P}{2}-\frac{3 \alpha_{\kappa}^{(1)} \Delta T}{2 a C_{\kappa}^{(1)}}\right)\left(C_{\kappa}^{(1)}-2 C_{\kappa}^{(3)}+\frac{2 D^{(1)}}{h}\right) \frac{\mu h}{2 a} \\
& +\frac{a^{2}}{B}\left(\frac{P}{2}-\frac{3 \alpha_{\kappa}^{(1)} \Delta T}{2 a C_{\kappa}^{(1)}}\right)^{2}\left(C_{\kappa}^{(1)}-2 C_{\kappa}^{(3)}+\frac{4 D^{(1)}}{h}+\frac{4 C_{\varepsilon}^{(1)}}{h^{2}}\right)\left(\frac{\mu h}{2 a}\right)^{2}
\end{aligned}
$$

where $G_{b}^{0}$ is the energy release rate in the absence of friction given by Eq. (39).

In the absence of residual stresses, the energy release rate is

$$
G_{b}=\frac{P^{2} a^{2}}{4 B}\left[\left(1-\frac{\mu h}{2 a}\right)^{2}\left(C_{\kappa}^{(1)}-2 C_{\kappa}^{(3)}\right)-\frac{4 D^{(1)}}{h} \frac{\mu h}{2 a}\left(1-\frac{\mu h}{2 a}\right)+\frac{4 C_{\varepsilon}^{(1)}}{h^{2}}\left(\frac{\mu h}{2 a}\right)^{2}\right]
$$

Finally, for homogeneous beams (where $D^{(1)}=0$ and $C_{\kappa}^{(i)}=1 /\left(E I^{(i)}\right)$ ), the result is

$$
G_{b}=\frac{9}{4} \frac{P^{2} a^{2}}{B^{2} E h^{3}}\left[1-2\left(\frac{\mu h}{2 a}\right)+\frac{13}{9}\left(\frac{\mu h}{2 a}\right)^{2}\right]
$$

These last two results are conditions where the arms have equal curvature and thus there may be contact along the entire crack surface. There were derived, however, as a limiting result of a point contact analysis. 


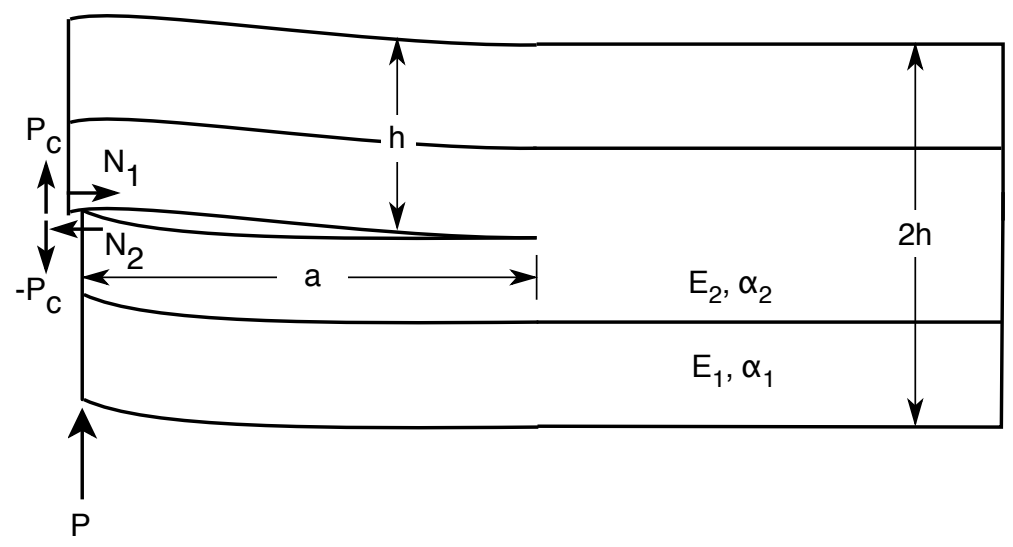

Fig. 3. Mode II loading of the adhesive specimen given in Fig. 2 when the crack is at the midplane. Friction forces will lead to equal and opposite normal forces $\left(N_{2}=-N_{1}\right)$ at the loading points with $n_{1}=0$ and $n_{2}=h_{2}=h$.

\subsection{ADHESIVE SPECIMEN NUMERICAL VERIFICATION}

In the absence of mechanical loads, the thermal energy release rates are given by Eq. (26) or Eq. (27) depending on whether or not there is contact at the arm ends. Figure 4 compares Eq. (26) for separated arms to finite element results for various values of $R$ and $\lambda$. The left side of the figure shows results as a function of $\lambda$ for constant $R=10$; the right side of the figure shows the results as a function of $R$ for constant $\lambda=1.5$; only half of each curve is shown because the results are symmetric about $\xi=0.5$. The calculated energy release rates were normalized by dividing by $E_{1} t_{1} \Delta \alpha^{2} \Delta T^{2}$. Expressions for the needed terms $\alpha_{\kappa}^{(i)}$ and $C_{\kappa}^{(i)}$ are given in Appendix A. The finite element analysis was done using JANFEA with 8-noded quadrilateral elements. ${ }^{9}$ Energy release rates were found using standard crack closure methods. ${ }^{10}$ Because bending deformations due to thermal stresses are quadratic the FEA analysis is very accurate even for crude meshes. The comparison shows that FEA and beam theory agree exactly; all results agreed to four or more significant figures. FEA results can partition total energy release rate into mode I and mode II. The thermal energy release rate is pure mode I when $\xi=0.5$. The mode II content increases as $\xi \rightarrow 1.0$ or $\xi \rightarrow 0.0$, eventually becoming larger than the mode I content. The energy release rate is a maximum for an interfacial crack suggesting a thermally-induced crack might tend toward either interface. On the other hand, the mode I energy release rate is a maximum for a crack at $\xi=0.5$. If the mode II toughness is higher than the mode I toughness, the crack might tend toward the middle of the adhesive.

When the ends of the arms are in contact, numerical verification requires a method that can deal with contact. Although FEA can handle contact with contact elements, here a new method called the material point method (MPM) was used which can handle contact without predefining contact surfaces. The development of MPM with explicit cracks is decribed in Refs. [11] and [12]. For these calculations, the previous contact algorithms ${ }^{11,13}$ were improved to be based on crack opening displacement rather than nodal volume or stress.

A comparison of MPM to theoretical results for a mode I adhesive specimen with a mid-plane crack $(\xi=0.5)$ and with $R=10, \lambda=3, t_{1}=9 \mathrm{~mm}, a=50 \mathrm{~mm}, \Delta \alpha=-6.0 \times 10^{-5} \mathrm{C}^{-1}$, and $\Delta T=-200^{\circ} \mathrm{C}$ is shown in Fig. 5. The loads were normalized to $P^{*}$ and the energy release rate was normalized by dividing by $E_{1} t_{1} \Delta \alpha^{2} \Delta T^{2}$. The theoretical results and $P^{*}$ are in Eq. (31). Below $P^{*}$, the energy release rate was constant and agreed precisely with $G_{b}$. Above $P^{*}$, the energy release rate increased but the numerical results were larger than $G_{a}$ in Eq. (31). This discrepancy, however, was not due to an error in the thermal energy release rates calculated here, but rather due to well-known, crack-tip rotation effects that are a consequence of shear deformation and the mechanical loads. ${ }^{14}$ Hence, Eq. (31) is nearly exact below $P^{*}$ where deformation is independent of mechanical loads, but is in error above $P^{*}$ where shear corrections are needed. Prior work on adhesive double cantilever beam specimens ${ }^{15,16}$ showed that beam theory can be corrected by replacing 


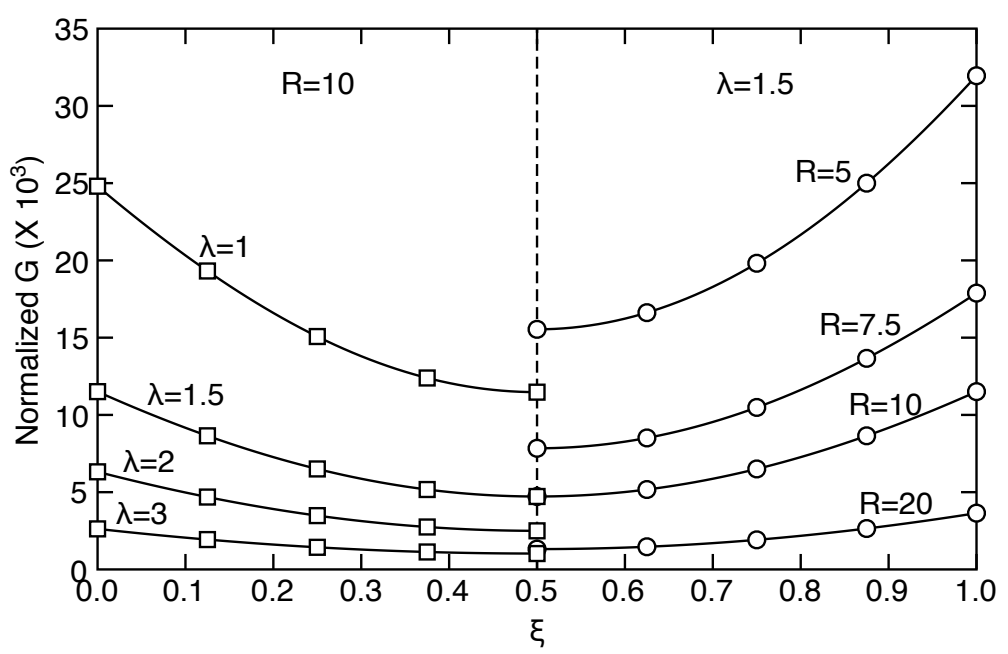

Fig. 4. A comparison of normalized energy release rate by finite element analysis results (symbols) to $G_{a}$ in Eq. (26) (curves) for non-contacting, adhesive specimens as a function of crack position. The left side varies $\lambda=t_{1} / t_{2}$ for fixed $R=10$, while the right side varies modulus ratio $R=E_{1} / E_{2}$ for fixed $\lambda=1.5$. The results are symmetric in crack position $\xi$; thus each side could be reflected about $\xi=0.5$ for full curves vs. $\xi$.

the actual crack length by an effective crack length of

$$
a_{\text {eff }}=a\left(1+\frac{\chi h_{1}}{a}\right) \quad \text { where } \quad \chi=1.15\left(\frac{1+\frac{R}{\lambda}}{6}\right)^{1 / 4}
$$

For these numerical calculations, $\chi h_{1} / a=0.19$ which is a rather large correction due to the small aspect ratio of the arms of the analyzed specimens $\left(a / h_{1}=4.17\right)$. The MPM results and corrected beam theory results agree well (see Fig. 5). Notice that corrected beam theory causes the critical load for loss of contact to be reduced to $0.84 P^{*}$. When the corrected contact load at low loads is substituted into the energy release rate expression, the correction factors cancel out and thus the energy release rate below the corrected $P^{*}$ is constant and equal to $G_{b}$ without need for any correction factor.

A comparison of MPM to theoretical results for a mode II adhesive specimen (the same geometry as for the mode I calculations) is shown in Fig. 6. The loads were normalized to $\left|P^{*}\right|$ and the energy release rate was normalized by dividing by $E_{1} t_{1} \Delta \alpha^{2} \Delta T^{2}$. The theoretical results and $P^{*}$ are in Eqs. (39) and (40). Compared to mode I, $P^{*}$ for mode II is negative and doubled in magnitude. Since all positive, applied loads are greater then $P^{*}$, the arms are always in contact. These first calculations assumed frictionless contact. When $P=0$ and energy release rate is due to residual stresses alone, the results agreed precisely with $G_{b}$. As load increased, the numerical results are close to, but higher than theoretical results. As in mode I results, the differences were attributed to shear deformation effects in the mechanical terms. ${ }^{14}$ The mechanical terms can again be corrected by replacing $a$ by $a_{e f f}$. Here the effective crack length was determined numerically by comparison of MPM results to beam theory. For this specific specimen, $a_{e f f}=1.048 a$. The numerically corrected beam theory fits MPM results well. There is slightly more variation in mode II MPM results than in mode I MPM results, which might be a consequence of the numerical difficulty of dealing with contact problems.

The contact methods in MPM can also model frictional contact. ${ }^{11,13} \mathrm{~A}$ comparison of MPM to theoretical results for the mode II specimen described above but with fixed $P=0.7\left|P^{*}\right|$ and variable friction is in Fig. 7 . The plot has the change in energy release rate or $G_{b}-G_{b}^{0}$. Friction causes the energy release rate to drop. Since normalized $G_{b}^{0}=6.45 \times 10^{-3}$, the effect of friction caused up to $25 \%$ decrease in energy release rate. The theoretical result with friction (see Eq. (45)) agrees well with numerical results. The dashed line in Fig. 7 plots only the linear term in Eq. (45). The linear term is accurate for low friction, but less accurate for higher friction. The numerical calculations used low-aspect-ratio arms with $a / h=4.17$. With higher aspect ratio arms, the linear term would be more accurate to higher friction. The linear term should suffice 


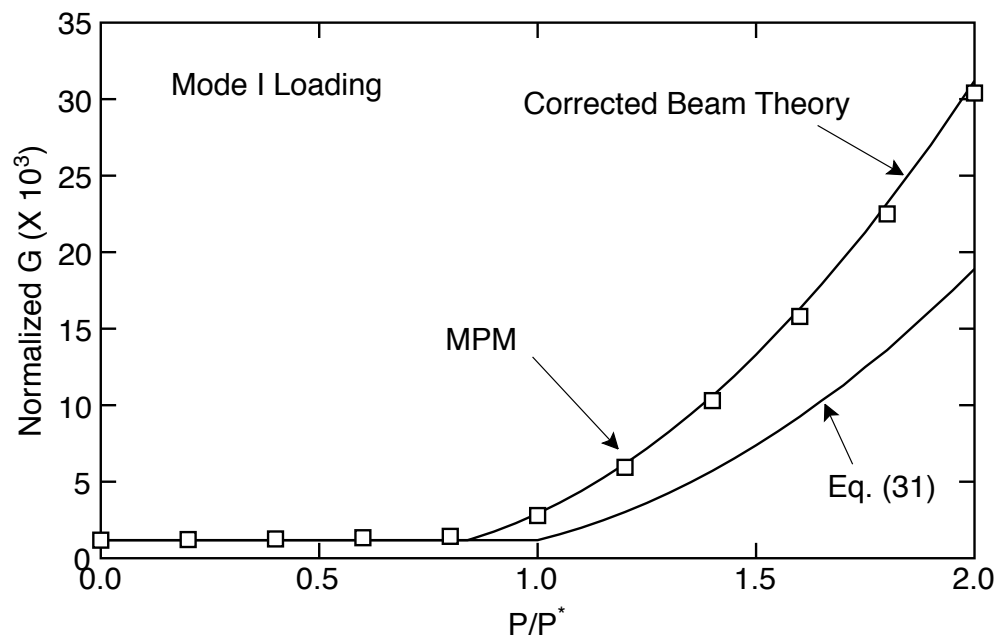

Fig. 5. A comparison of normalized energy release rate by material point method (symbols) to calculated energy release rates (curves) for a mode I loaded specimen with a midplane crack $(\xi=0.5)$ as a function of normalized applied load. The curve labeled "Eq. (31)" is the uncorrected result; the curve labeled "Corrected Beam Theory" corrects the mechanical loading terms for shear deformation effects.

for all friction conditions provided $a / h>10$. Otherwise the quadratic term can be included for greater accuracy.

\subsection{Single LEG Bend TEST FOR INTERFACIAL FRACTURE}

A particularly simple example, because the two arms are homogeneous and residual stresses do not cause contact (at least in beam theory approximations), is interface fracture in the single-leg bending specimen ${ }^{17}$ as illustrated in Fig. 8. For this specimen, $\alpha_{\kappa}^{(1)}={\sigma_{\alpha E}^{(1)}}^{2}=\alpha_{\kappa}^{(2)}={\sigma_{\alpha E}^{(2)}}^{2}=0$, but $\alpha_{\kappa}^{(3)} \neq 0$ and

$$
h_{3} E_{0}^{(3)}{\sigma_{\alpha E}^{(3)}}^{2}=\frac{E_{1} t_{1} \Delta \alpha^{2}}{1+R \lambda}
$$

For a crack of length $a<L, M_{1}=M_{3}=P a / 2$ and $M_{2}=N_{i}=0$. Substitution into Eq. (12) gives

$$
G=\frac{P^{2} a^{2}}{8 B}\left(C_{\kappa}^{(1)}-C_{\kappa}^{(3)}\right)-\frac{P a \alpha_{\kappa}^{(3)} \Delta T}{2 B}-\frac{\alpha_{\kappa}^{(3)^{2}} \Delta T^{2}}{2 B C_{\kappa}^{(3)}}+\frac{E_{1} t_{1} \Delta \alpha^{2} \Delta T^{2}}{2(1+R \lambda)}
$$

In the absence of residual stresses, this result reduces to the results in Ref. [17] that ignored residual stresses.

In the absence of mechanical loads and substituting the two-layer results in Appendix A for $\alpha_{\kappa}^{(3)}$ and $C_{\kappa}^{(3)}$, the energy release rate for spontaneous adhesive failure is

$$
G=\frac{1}{2} \frac{E_{1} t_{1} \Delta \alpha^{2} \Delta T^{2}\left(1+R \lambda^{3}\right)}{1+R \lambda(4+\lambda(6+\lambda(4+R \lambda)))}
$$

This result is independent of crack length and specimen size. It is thus the appropriate equation to predict spontaneous adhesive failure of any two layer composite. If the interfacial toughness, $G_{c}$, is less then $G$, then the adhesive bond will fail due to residual stresses alone. For example, recent experiments on PE/steel bonds had some results that delaminated after aging. The delamination occurred because aging caused the interfacial toughness to drop such that $G_{c}<G .{ }^{18}$

For numerical verification of this thermal-load-only result, Fig. 9 plots normalized energy release rate (or $\left.G /\left(E_{t} t_{1} \Delta \alpha^{2} \Delta T^{2}\right)\right)$ as a function of modulus ratio $R$ for various values of $\lambda$. There is an unusual affect of $\lambda$ on the $R$-dependence of energy release rate, but again, beam theory analysis in Eq. (51) gives essentially exact results for energy release rate due to residual stresses alone. 


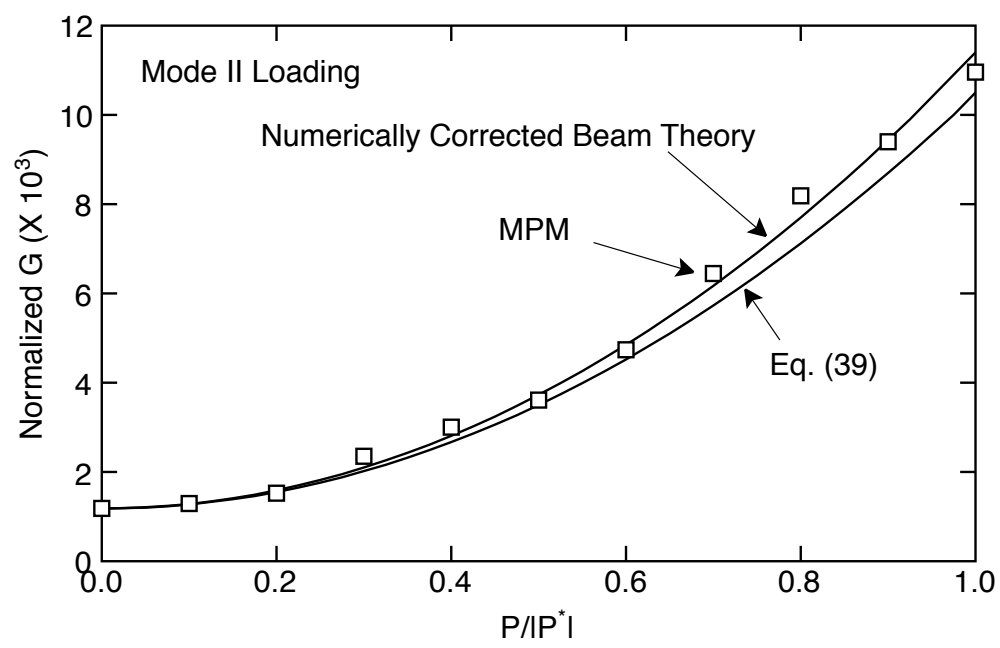

Fig. 6. A comparison of normalized energy release rate by material point method (symbols) to calculated energy release rates (curves) for a mode II loaded specimen with a midplane crack $(\xi=0.5)$ as a function of normalized applied load. The curve labeled "Eq. (39)" is the uncorrected result; the curve labeled "Numerically Corrected Beam Theory" corrects the mechanical loading terms for shear deformation effects.

\subsection{SKIN-CORE INTERFACIAL FRACTURE IN SANDWICH LAMINATES}

A three point bending specimen with an initial crack between the skin and the core of a skin-core, sandwich composite has been used to study skin-core adhesion ${ }^{19}$ (see Fig. 10). Skin-core composites are often made by curing the skins and then gluing them to the core. A full analysis thus requires the five layers shown in Fig. 10 - two skin layers with modulus, thermal expansion coefficient and thickness of $E_{s}, \alpha_{s}$, and $t_{s}$, a core layer with $E_{c}, \alpha_{c}$, and $t_{c}$, and two adhesive layers with $E_{a}, \alpha_{a}$, and $t_{a}$. All layers are assumed here to be homogeneous, or if the skins are laminae, they are assumed to be symmetric laminae where $E_{s}$ and $\alpha_{s}$ are the axial properties of the skins. It would be easy to account for unsymmetric laminate skins by including the layers of the skin laminae as separate layers in the analysis. The adhesive layer is shown with dotted lines because common core materials are porous (e.g., honeycomb, foam, or wood). When the core is porous, $t_{a}$ is the depth of penetration of the adhesive into the core and $E_{a}$ and $\alpha_{a}$ are the effective properties of the adhesive/core composite that results near the interface.

Figure 10 shows a specimen with a crack between the top skin (arm 1) and remainder of the structure (four-layers with adhesive/core/adhesive/lower skin as arm 2). If the crack diverts to the adhesive/core interface, then the top skin plus adhesive would be arm 1 while the remaining three layers (core/adhesive/lower skin) would be arm 2. At this point, the only difference between a crack below the skin and a crack between the adhesive and the core is that $\alpha_{\kappa}^{(1)}=0$ in the former, but $\alpha_{\kappa}^{(1)} \neq 0$ in the later. For a general analysis, $\alpha_{\kappa}^{(1)}$ is included in the analysis. Figure 10 shows arm 2 bending toward arm 1, which implies $\left(\alpha_{\kappa}^{(1)}-\alpha_{\kappa}^{(2)}\right) \Delta T<0$. When contact occurs, there will be a positive contact force $P_{c}$ on arm 1 and compensating force $-P_{c}$ on arm 2 located at the contact position of $x=b$. Assuming the crack is to the left of the center load $(a<L / 2)$, the moment-curvature relations on the two arms for $x>b$ where the load point is at $x=0$ are:

$$
\frac{d^{2} v^{(1)}}{d x^{2}}=C_{\kappa}^{(1)}\left(\frac{P x}{2}+P_{c}(x-b)\right)+\alpha_{\kappa}^{(1)} \Delta T \quad \frac{d^{2} v^{(2)}}{d x^{2}}=-C_{\kappa}^{(2)} P_{c}(x-b)+\alpha_{\kappa}^{(2)} \Delta T
$$

Integrating to find displacement, the contact force required to obtain zero crack opening displacement at $x=b$ is found to be

$$
P_{c}=-\frac{3\left(\alpha_{\kappa}^{(1)}-\alpha_{\kappa}^{(2)}\right) \Delta T}{2(a-b)\left(C_{\kappa}^{(1)}+C_{\kappa}^{(2)}\right)}-\frac{C_{\kappa}^{(1)}}{C_{\kappa}^{(1)}+C_{\kappa}^{(2)}}\left(\frac{2 a+b}{2(a-b)}\right) \frac{P}{2}
$$

There are thus two regimes. When the load is low, there will be contact at $x=b$ and $P_{c}$ will be positive. The contact regime ends when the applied load causes separation which occurs when $P_{c}$ in Eq. (53) drops 


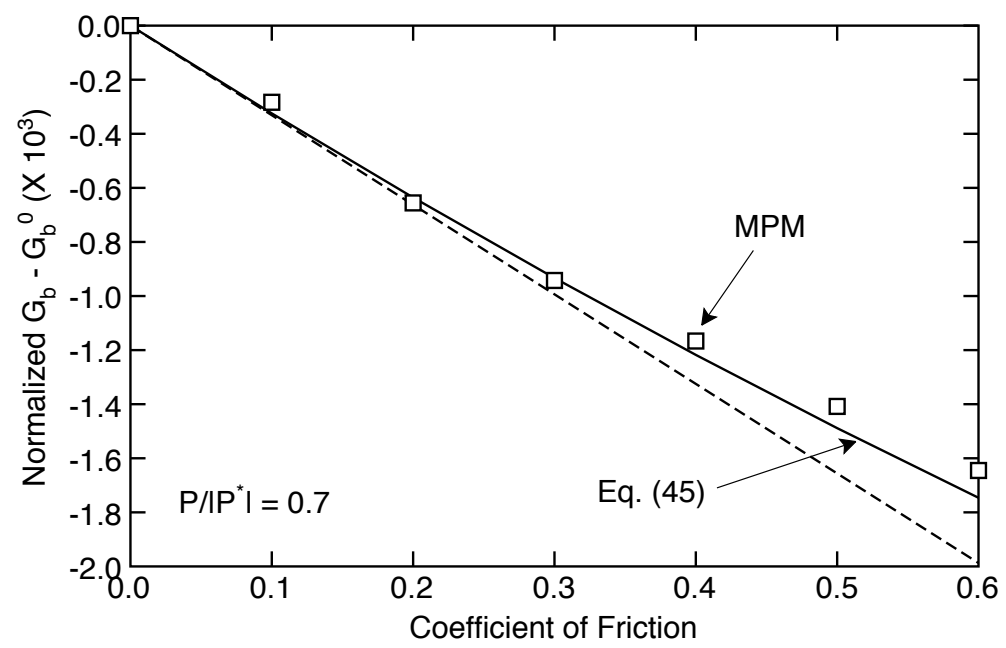

Fig. 7. A comparison of change in normalized energy release rate due to friction calculated by material point method (symbols) to energy release rate in Eq. (45) (curve) for a mode II loaded specimen with a midplane crack $(\xi=0.5)$ as a function of friction coefficient. The load was constant at $P=0.7\left|P^{*}\right|$. The curve labeled "Eq. (45)" is the full analysis; the dashed curve includes only the first, or linear, term in Eq. (45).

to zero. In other words

$$
\begin{array}{cc}
\text { Contact Regime : } & 0<P<-\frac{6\left(\alpha_{\kappa}^{(1)}-\alpha_{\kappa}^{(2)}\right) \Delta T}{(2 a+b) C_{\kappa}^{(1)}} \\
\text { Separation Regime : } & P>-\frac{6\left(\alpha_{\kappa}^{(1)}-\alpha_{\kappa}^{(2)}\right) \Delta T}{(2 a+b) C_{\kappa}^{(1)}}
\end{array}
$$

Negative $P$ is not considered because three-point bending is limited to positive $P$. Furthermore, if $\left(\alpha_{\kappa}^{(1)}-\right.$ $\left.\alpha_{\kappa}^{(2)}\right) \Delta T>0$, the contact regime is absent and all loads result in separation.

In the contact regime

$$
\alpha_{\kappa}^{(3)}=0, \quad N_{i}=0, \quad M_{1}=\frac{P a}{2}+P_{c}(a-b), \quad M_{2}=-P_{c}(a-b), \quad M_{3}=\frac{P a}{2}
$$

which leads to

$$
\begin{aligned}
G_{c o n}= & \frac{1}{2 B}\left[\frac{P^{2} a^{4}}{4}\left(C_{\kappa}^{(1)}-C_{\kappa}^{(3)}\right)+P_{c}(a-b)\left(P a C_{\kappa}^{(1)}+2\left(\alpha_{\kappa}^{(1)}-\alpha_{\kappa}^{(2)}\right) \Delta T+P_{c}(a-b)\left(C_{\kappa}^{(1)}+C_{\kappa}^{(2)}\right)\right)\right. \\
& \left.+P a \alpha_{\kappa}^{(1)} \Delta T\right]+\frac{\Delta T^{2}}{2 B}\left(\frac{\alpha_{\kappa}^{(1)}{ }^{2}}{C_{\kappa}^{(1)}}+\frac{\alpha_{\kappa}^{(2)}}{C_{\kappa}^{(2)}}-B h_{1} E_{0}^{(1)} \sigma_{\alpha E}^{(1)}-B h_{2} E_{0}^{(2)}{\sigma_{\alpha E}^{(2)}}^{2}+B h_{3} E_{0}^{(3)} \sigma_{\alpha E}^{(3)^{2}}\right)
\end{aligned}
$$

In the separation regime

$$
\alpha_{\kappa}^{(3)}=0, \quad N_{i}=0, \quad M_{1}=\frac{P a}{2}, \quad M_{2}=0, \quad M_{3}=\frac{P a}{2}
$$

which leads to

$$
\begin{aligned}
G_{\text {sep }}=\frac{P^{2} a^{2}}{8 B} & \left(C_{\kappa}^{(1)}-C_{\kappa}^{(3)}\right)+\frac{P a \alpha_{\kappa}^{(1)} \Delta T}{2 B} \\
& +\frac{\Delta T^{2}}{2 B}\left(\frac{\alpha_{\kappa}^{(1)}{ }^{2}}{C_{\kappa}^{(1)}}+\frac{\alpha_{\kappa}^{(2)^{2}}}{C_{\kappa}^{(2)}}-B h_{1} E_{0}^{(1)}{\sigma_{\alpha E}^{(1)}}^{2}-B h_{2} E_{0}^{(2)}{\sigma_{\alpha E}^{(2)}}^{2}+B h_{3} E_{0}^{(3)}{\sigma_{\alpha E}^{(3)}}^{2}\right)
\end{aligned}
$$




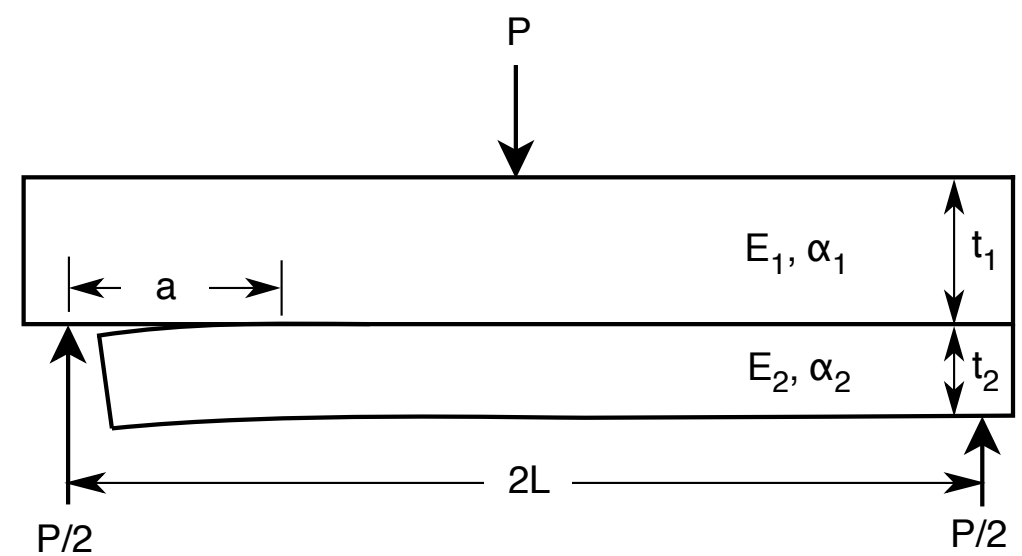

Fig. 8. A single-leg bending test specimen with an interfacial crack. The two layers have moduli, thermal expansion coefficients, and thicknesses of $E_{1}, \alpha_{1}$, and $t_{1}$ or $E_{2}, \alpha_{2}$, and $t_{2}$. The specimen is loaded in three-point bending.

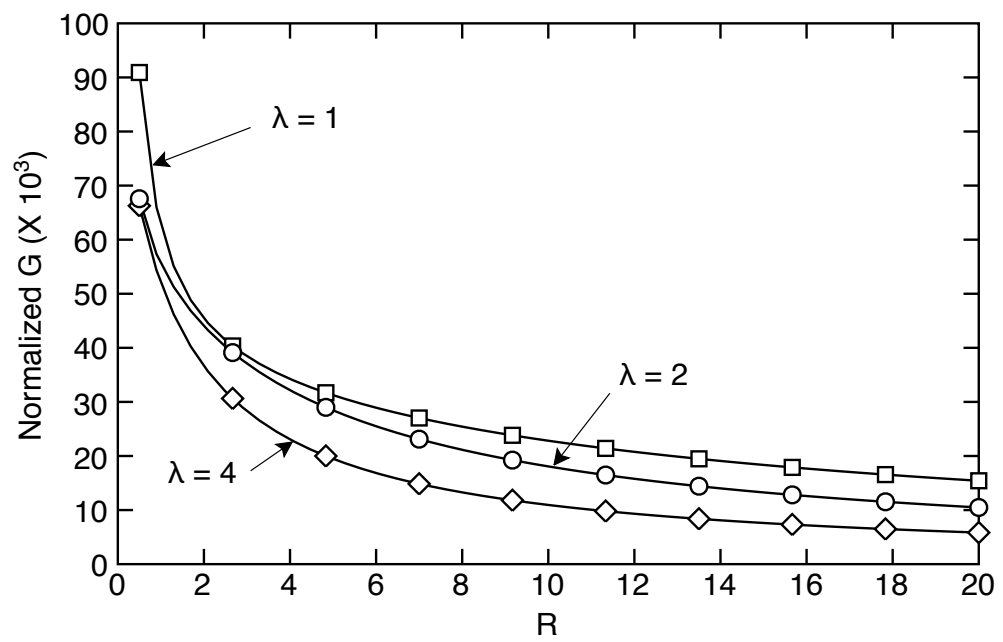

Fig. 9. A comparison of normalized energy release rate by finite element analysis results (symbols) to $G$ in Eq. (51) (curves) for single-leg bending specimens as a function of modulus ratio $R=E_{1} / E_{2}$ for various values of $\lambda=t_{1} / t_{2}$.

When $\left(\alpha_{\kappa}^{(1)}-\alpha_{\kappa}^{(2)}\right) \Delta T>0$, the contact region is absent and $G$ will be given by $G_{s e p}$ for all $P \geq 0$.

Further simplification (such as for the ${\sigma_{\alpha E}^{(i)}}^{2}$ terms) requires specification of the location of the crack. When the crack is between the top skin and the adhesive (denoted as $s a$ ), $\alpha_{\kappa}^{(1)}=\sigma_{\alpha E}^{(1)}{ }^{2}=0$. Substituting $P_{c}$ in Eq. (53) into Eq. (57) and using Eq. (95) in Appendix A, the final result is

$$
\begin{aligned}
G_{c o n}^{(s a)}= & \frac{P^{2}}{32 B} \frac{4 a^{2}\left(C_{\kappa}^{(1)} C_{\kappa}^{(2)}-\left(C_{\kappa}^{(1)}+C_{\kappa}^{(2)}\right) C_{\kappa}^{(3)}\right)+b^{2} C_{\kappa}^{(1)}{ }^{2}}{C_{\kappa}^{(1)}+C_{\kappa}^{(2)}}+\frac{P \Delta T}{8 B} \frac{(4 a-b) C_{\kappa}^{(1)} \alpha_{\kappa}^{(2)}}{C_{\kappa}^{(1)}+C_{\kappa}^{(2)}} \\
& +\frac{\Delta T^{2}}{2 B}\left(\frac{\alpha_{\kappa}^{(2)}{ }^{2}}{C_{\kappa}^{(2)}} \frac{4 C_{\kappa}^{(1)}+C_{\kappa}^{(2)}}{4\left(C_{\kappa}^{(1)}+C_{\kappa}^{(2)}\right)}+B E_{s} t_{s} \frac{\left(2 R_{a}+R_{c}\right)\left(2 R_{a} \Delta \alpha_{a s}^{2}+R_{c} \Delta \alpha_{c s}^{2}\right)-2 R_{a} R_{c} \Delta \alpha_{a c}^{2}}{\left(1+2 R_{a}+R_{c}\right)\left(2+2 R_{a}+R_{c}\right)}\right)
\end{aligned}
$$

where $R_{a}=E_{a} t_{a} /\left(E_{s} t_{s}\right), R_{c}=E_{c} t_{c} /\left(E_{s} t_{s}\right)$, and $\Delta \alpha_{i j}=\alpha_{i}-\alpha_{j}$. In the separation regime, the skin/adhesive crack result reduces to

$$
G_{\text {sep }}^{(s a)}=\frac{P^{2} a^{2}}{8 B}\left(C_{\kappa}^{(1)}-C_{\kappa}^{(3)}\right)+\frac{\Delta T^{2}}{2 B}\left(\frac{\alpha_{\kappa}^{(2)^{2}}}{C_{\kappa}^{(2)}}+B E_{s} t_{s} \frac{\left(2 R_{a}+R_{c}\right)\left(2 R_{a} \Delta \alpha_{a s}^{2}+R_{c} \Delta \alpha_{c s}^{2}\right)-2 R_{a} R_{c} \Delta \alpha_{a c}^{2}}{\left(1+2 R_{a}+R_{c}\right)\left(2+2 R_{a}+R_{c}\right)}\right)
$$




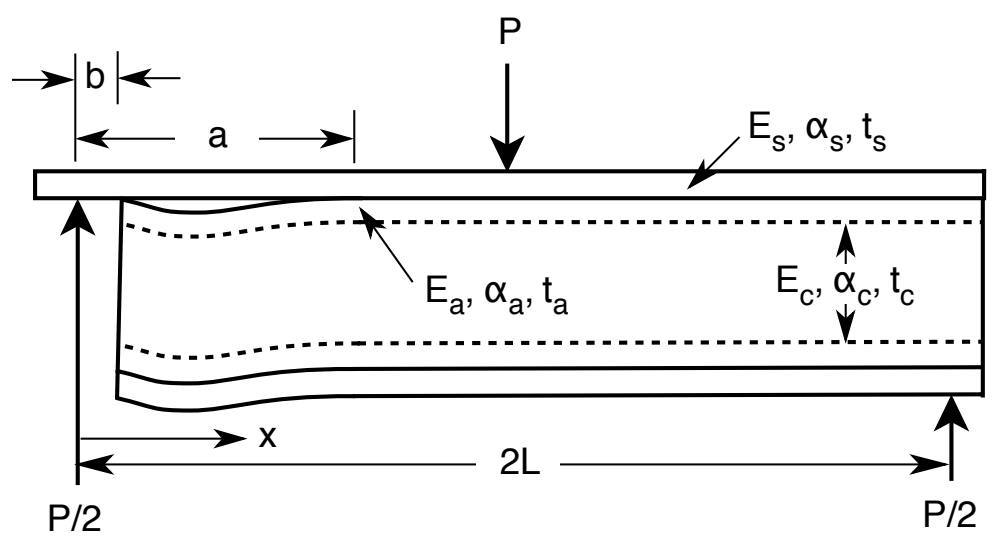

Fig. 10. A three-point bending specimen used to study adhesion between the skin and the core of a sandwich composite structure. The skin, adhesive layer, and core have moduli, thermal expansion coefficients, and thicknesses of $E_{s}, \alpha_{s}$, and $t_{s}, E_{a}, \alpha_{a}$, and $t_{a}$, or $E_{c}, \alpha_{c}$, and $t_{c}$, respectively. This figure shows a crack between the skin and the adhesive. In some tests, the crack diverts to the interface between the adhesive and the core.

If the crack diverts to the adhesive/core interface (denoted as $a c$ ), $\alpha_{\kappa}^{(1)} \neq 0$ and ${\sigma_{\alpha E}^{(1)}}^{2} \neq 0$. Substituting $P_{c}$ in Eq. (53) into Eq. (57) and using Eq. (95) in Appendix A, the final result is

$$
\begin{aligned}
G_{\text {con }}^{(a c)}=\frac{P^{2}}{32 B} & \frac{\left.4 a^{2}\left(C_{\kappa}^{(1)} C_{\kappa}^{(2)}-\left(C_{\kappa}^{(1)}+C_{\kappa}^{(2)}\right) C_{\kappa}^{(3)}\right)+b^{2} C_{\kappa}^{(1)}\right)^{2}}{C_{\kappa}^{(1)}+C_{\kappa}^{(2)}} \\
& +\frac{P \Delta T}{8 B} \frac{4 a\left(C_{\kappa}^{(2)} \alpha_{\kappa}^{(1)}+C_{\kappa}^{(1)} \alpha_{\kappa}^{(2)}\right)+b\left(\alpha_{\kappa}^{(1)}-\alpha_{\kappa}^{(2)}\right) C_{\kappa}^{(1)}}{C_{\kappa}^{(1)}+C_{\kappa}^{(2)}} \\
& +\frac{\Delta T^{2}}{2 B}\left[\frac{\alpha_{\kappa}^{(1)}}{C_{\kappa}^{(1)}} \frac{C_{\kappa}^{(1)}+4 C_{\kappa}^{(2)}}{4\left(C_{\kappa}^{(1)}+C_{\kappa}^{(2)}\right)}+\frac{\alpha_{\kappa}^{(2)}}{C_{\kappa}^{(2)}} \frac{4 C_{\kappa}^{(1)}+C_{\kappa}^{(2)}}{4\left(C_{\kappa}^{(1)}+C_{\kappa}^{(2)}\right)}\right. \\
& \left.+B E_{s} t_{s} \frac{\left(\left(1+R_{a}\right)\left(R_{a} \alpha_{a c}^{2}+\alpha_{c s}^{2}\right)-R_{a} \alpha_{a s}^{2}\right) R_{c}^{2}}{\left(1+R_{a}\right)\left(1+R_{a}+R_{c}\right)\left(2+2 R_{a}+R_{c}\right)}\right]
\end{aligned}
$$

In the separation regime, the adhesive/core crack result reduces to

$$
\begin{aligned}
G_{\text {sep }}^{(a c)}=\frac{P^{2} a^{2}}{8 B} & \left(C_{\kappa}^{(1)}-C_{\kappa}^{(3)}\right)+\frac{P a \alpha_{\kappa}^{(1)} \Delta T}{2 B} \\
& +\frac{\Delta T^{2}}{2 B}\left(\frac{\alpha_{\kappa}^{(1)}{ }^{2}}{C_{\kappa}^{(1)}}+\frac{\alpha_{\kappa}^{(2)^{2}}}{C_{\kappa}^{(2)}}+B E_{s} t_{s} \frac{\left(\left(1+R_{a}\right)\left(R_{a} \alpha_{a c}^{2}+\alpha_{c s}^{2}\right)-R_{a} \alpha_{a s}^{2}\right) R_{c}^{2}}{\left(1+R_{a}\right)\left(1+R_{a}+R_{c}\right)\left(2+2 R_{a}+R_{c}\right)}\right)
\end{aligned}
$$

For numerical verification of the non-contact, thermal-load-only results, an analysis was done for a composite sandwich construction. The skins had typical laminate properties of $E_{s}=70 \mathrm{GPa}, \nu_{s}=0.25$, $\alpha_{s}=2 \times 10^{-6} \mathrm{C}^{-1}$, and $t_{s}=1 \mathrm{~mm}$. The adhesive had typical properties of $E_{a}=3.5 \mathrm{GPa}, \nu_{a}=0.3$, $\alpha_{a}=100 \times 10^{-6} \mathrm{C}^{-1}$, and $t_{a}=0.6667 \mathrm{~mm}$. The thicker core had $\nu_{c}=0.2, \alpha_{c}=40 \times 10^{-6} \mathrm{C}^{-1}$, and $t_{c}=20 \mathrm{~mm}$. The modulus of the core was varied from $E_{c}=3.5 \mathrm{MPa}$ to $E_{c}=3.5 \mathrm{GPa}$ such that $R_{c}$ varied from 0.001 to 1 . The specimen thickness was $B=1 \mathrm{~mm}$ (although thermal energy release rate is independent of $B$ ) and temperature was $\Delta T=200^{\circ} \mathrm{C}$. The results for (unnormalized) energy release rate as a function of $R_{c}$ for both a skin/adhesive crack and an adhesive/core crack are in Fig. 11. The finite element results (symbols) agree nearly exactly with Eqs. (61) and (63). The energy release rate for the adhesive/core crack is lower than for the skin/adhesive crack. The skin/adhesive crack was close to pure mode II. The adhesive/core crack had much more mode I component

Recent experiments looked at the affect of residual stresses on failure of sandwich/core composites with carbon-fiber/bismaleimide (BMI) skins combined with either aluminum honeycomb or carbon foam as the 


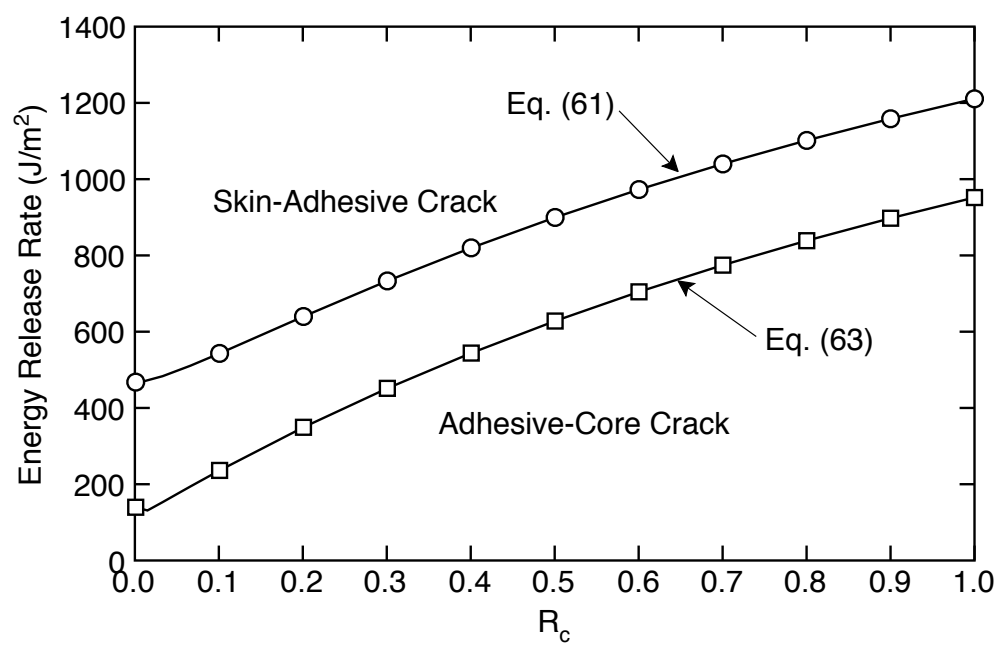

Fig. 11. A comparison of total energy release rate by finite element analysis results (symbols) to energy release rates in Eqs. (61) and (63) (curves) for a sandwich laminate as a function of skin/core stiffness ratio $R_{c}=E_{c} t_{c} /\left(E_{s} t_{s}\right)$. The two curves are for a crack between the skin and the adhesive or a crack between the adhesive and the core. See text for details on the properties of the layers.

core. $^{20}$ The hypothesis was that because carbon foam has a lower thermal expansion coefficient than aluminum honeycomb, the better match to the composite skins might provide advantages in high-temperature applications. The experiments used the geometry in Fig. 10. The aluminum honeycomb specimens failed at the skin/adhesive interface and were analyzed using Eq. (61). Calculations showed that the energy release rate due to thermal stresses was larger than the energy release rate due to mechanical loads at failure. Thus residual stresses play an important role in the failure of aluminum honeycomb sandwich composites. In contrast, the carbon foam specimens failed at the adhesive/core interface and thus were analyzed using Eq. (63). Calculations showed that the better match in thermal expansion coefficient did eliminate residual stress effects in the carbon foam specimens. Unfortunately, the inherent toughness of the carbon foam is low and thus performance of the carbon foam specimens was worse than the aluminum honeycomb specimens at all temperatures. It was also observed that differential shrinkage between the adhesive that penetrates into the foam caused cracks in the foam. These cracks caused the adhesive-core interface crack to have lower toughness than pure carbon foam.

\subsection{Longitudinal SPlitting}

When highly anisotropic materials, such as wood or unidirectional composites, are loaded in tension parallel to the fibers or bending transverse to the fibers (see Fig. 12), failure is by longitudinal splitting rather than by self-similar crack growth. ${ }^{21-23}$ Similarly, a multilayered specimen with a crack at the interface between two layers may fail by splitting if the interface toughness is lower than the layer toughness. This section considers longitudinal splitting in a three-layered specimen with a crack at the interface and ignores contact which applies whenever $\alpha_{\kappa}^{(1)} \Delta T>0$. For the tension specimen where arm 1 is the intact core and opposite face, arm 2 is the face layer that has split off, and arm 3 is the entire specimen:

$$
N_{1}=N_{3}=P, \quad N_{2}=0, \quad M_{1}=M_{3}=\frac{P t_{1}}{2}, \quad M_{2}=0, \quad n_{1}=\frac{t_{2}}{2}, \quad \text { and } \quad n_{3}=t_{1}+\frac{t_{2}}{2}
$$

For a symmetric three-layer beam with a crack at the interface

$$
\alpha_{\kappa}^{(2)}={\sigma_{\alpha E}^{(2)}}^{2}=\alpha_{\kappa}^{(3)}=D^{(3)}=0
$$

The energy release rate for tensile loading is thus

$$
G_{T}=\frac{P^{2}}{8 B}\left(\left(C_{\kappa}^{(1)}-C_{\kappa}^{(3)}\right) t_{1}^{2}+4\left(C_{\varepsilon}^{(1)}-C_{\varepsilon}^{(3)}+D^{(1)} t_{1}\right)\right)+\frac{P \Delta T}{2 B}\left(\alpha_{\kappa}^{(1)} t_{1}+2\left(\alpha_{\varepsilon}^{(1)}-\alpha_{\varepsilon}^{(3)}\right)\right)
$$



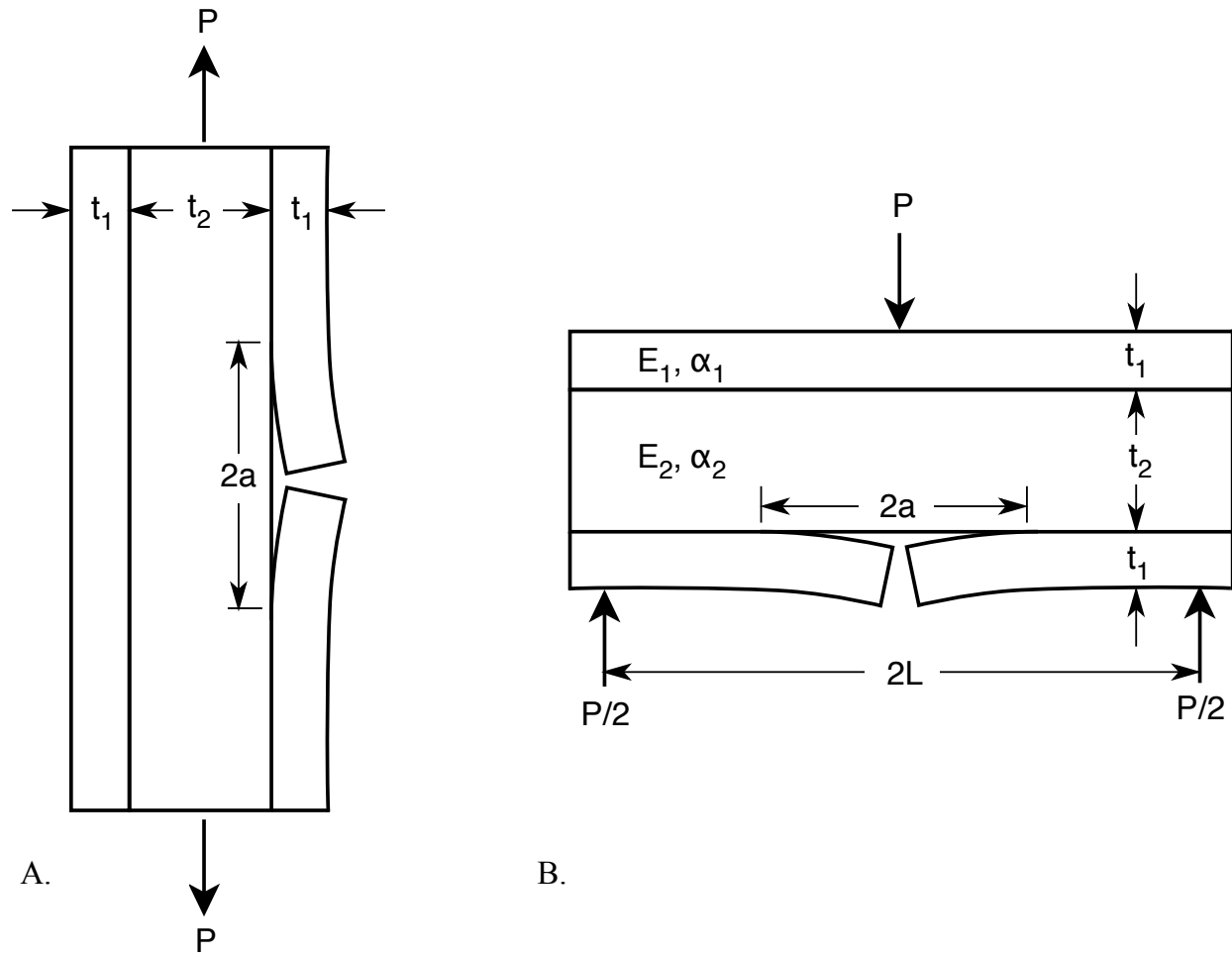

B.

Fig. 12. Longitudinal splitting at the interface between a surface layer and the central layer in symmetric three-layer specimens loading either in A. tension or B. three point bending. The surface and central layers have moduli, thermal expansion coefficients, and thicknesses of $E_{1}, \alpha_{1}$, and $t_{1}$ or $E_{2}, \alpha_{2}$, and $t_{2}$. The total split length is $2 a$. The span in the bending geometry is $2 L$.

$$
+\frac{\Delta T^{2}}{2 B}\left[\frac{\alpha_{\kappa}^{(1)^{2}}}{C_{\kappa}^{(1)}}+\frac{B E_{1} t_{1} \Delta \alpha^{2}}{(1+2 R \lambda)(1+R \lambda)}\right]
$$

As expected, the $\Delta T^{2}$ term is identical to the non-contact, end-loaded adhesive specimen result in Eq. (26) for the special case of $\xi=1$ and $\alpha_{\kappa}^{(2)}=0$. Note that $G_{T}$ is independent of split length $a$.

For the bending specimen

$$
M_{1}=M_{3}=\frac{P}{4}(L-a), \quad M_{2}=0, \quad \text { and } \quad N_{i}=0
$$

The energy release rate for bending is thus

$$
G_{B}=\frac{P^{2}(L-a)^{2}}{32 B}\left(C_{\kappa}^{(1)}-C_{\kappa}^{(3)}\right)+\frac{P(L-a) \alpha_{\kappa}^{(1)} \Delta T}{4 B}+\frac{\Delta T^{2}}{2 B}\left[\frac{\alpha_{\kappa}^{(1)^{2}}}{C_{\kappa}^{(1)}}+\frac{B E_{1} t_{1} \Delta \alpha^{2}}{(1+2 R \lambda)(1+R \lambda)}\right]
$$

Of course the $\Delta T^{2}$ terms are identical for tension and bending. The total energy release rate for bending, however, now depends on the split length $a$.

In the absence of residual stresses and following Williams, ${ }^{3}$ the energy release rates can be cast in fracture mechanics forms as

$$
G_{T}=\frac{\sigma_{T}^{2} t_{1}}{E_{c}} Y_{T}^{2} \quad \text { and } \quad G_{B}=\frac{\sigma_{B}^{2} t_{1}}{E_{c}} Y_{B}^{2}
$$

where tensile stress, $\sigma_{T}$, and tensile calibration function, $Y_{T}^{2}$, are

$$
\sigma_{T}=\frac{P}{2 B h} \quad \text { and } \quad Y_{T}^{2}=\frac{B E_{c} h^{2}}{2 t_{1}}\left(\left(C_{\kappa}^{(1)}-C_{\kappa}^{(3)}\right) t_{1}^{2}+4\left(C_{\varepsilon}^{(1)}-C_{\varepsilon}^{(3)}+D^{(1)} t_{1}\right)\right)
$$


The local bending stress, ${ }^{3} \sigma_{B}$, and the bending calibration function, $Y_{B}^{2}$, are

$$
\sigma_{B}=\frac{3 P(L-a)}{8 B h^{2}} \quad \text { and } \quad Y_{B}^{2}=\frac{2 B E_{c} h^{4}}{9 t_{1}}\left(C_{\kappa}^{(1)}-C_{\kappa}^{(3)}\right)
$$

The normalizing modulus was choose to be axial modulus of the structure or

$$
E_{c}=\frac{E_{1}}{R}\left(\frac{1+2 R \lambda}{1+2 \lambda}\right)
$$

For homogeneous beams, these calibration functions reduce to

$$
\begin{aligned}
Y_{T}^{2} & =\frac{1-2 \frac{t_{1}}{W}+10\left(\frac{t_{1}}{W}\right)^{2}-9\left(\frac{t_{1}}{W}\right)^{3}+3\left(\frac{t_{1}}{W}\right)^{4}}{2\left(1-\frac{t_{1}}{W}\right)^{3}} \\
Y_{B}^{2} & =\frac{3-3 \frac{t_{1}}{W}+\left(\frac{t_{1}}{W}\right)^{2}}{6\left(1-\frac{t_{1}}{W}\right)^{3}}
\end{aligned}
$$

These results agree with the Williams results (i.e., $Y_{T}^{2}=Y_{I T}^{2}+Y_{I I T}^{2}$ from Ref. [3], and $Y_{B}^{2}=Y_{I B}^{2}+Y_{I I B}^{2}$ from Ref. [3]). The new results in Eqs. (70) and (71) extend the Williams results to heterogeneous beams. The results in Eqs. (66) and (68) further extend the analysis to heterogeneous beams with residual stresses.

Numerical verification of the thermal-only energy release rate is not needed, because the results for longitudinal splitting specimens are a special case of adhesive specimen results in the "End-Loaded Adhesive Specimen" section. Those results were verified by comparison to FEA. For illustration, Fig. 13 plots the effect of heterogeneity on the calibration factors for splitting of a coating off a core under tensile or bending loads (see Eqs. (70) and (71)) in the absence of residual stresses. The results are for $R=E_{1} / E_{2} \geq 1$ or for a stiffer coating on a compliant core. Under bending loading, the energy release rate increases with $R$ make coating failure by splitting more likely. For tensile loading, the energy release rate decreases with $R$ for thin coatings, but increases for thicker coatings. In fact the energy release rate becomes negative for very thin coatings suggesting that thin, stiff layers will not split from cracks at an interface with a compliant layer due to mechanical energy release rate alone. Thermal stresses, however, will contribute positive energy release rate that may cause splitting. The plot stops at $t_{1} / W=0.5$ because that is the limit of $t_{1}$ for a three-layer system. The results for $t_{1} / W=0.5$ are independent of $R$ because layer 2 is absent and thus the specimen becomes homogeneous.

\section{Conclusions}

Equation (12) is a general energy release rate result for a large number of two-dimensional fracture problems in cracked laminates or cracked multi-layered structures. It extends prior energy release rate analyses ${ }^{1-3}$ to account for heterogeneous structures and to account for residual stresses. The total energy release rate including residual stresses can be found simply by analyzing any specific structure and resolving crack-tip normal forces and bending moments. The issue of partitioning total energy release rate into mode I and mode II components was not analyzed and may require numerical methods. ${ }^{1}$ All numerical results show that the thermal-stress part of the energy release rate in this analysis is essentially exact. The energy release rate component due to mechanical loads may require correction for shear-deformation effects; methods for that task are been described by others (e.g., Ref. [14]).

\section{Appendix A}

The effective properties of the three sublaminates in Fig. 1 can be determined from a laminated beam analysis. For a laminated beam under axial strain $\varepsilon_{0}$ and curvature $\kappa$, the axial strain as a function of $y$ is

$$
\varepsilon(y)=\varepsilon_{0}-\kappa y
$$




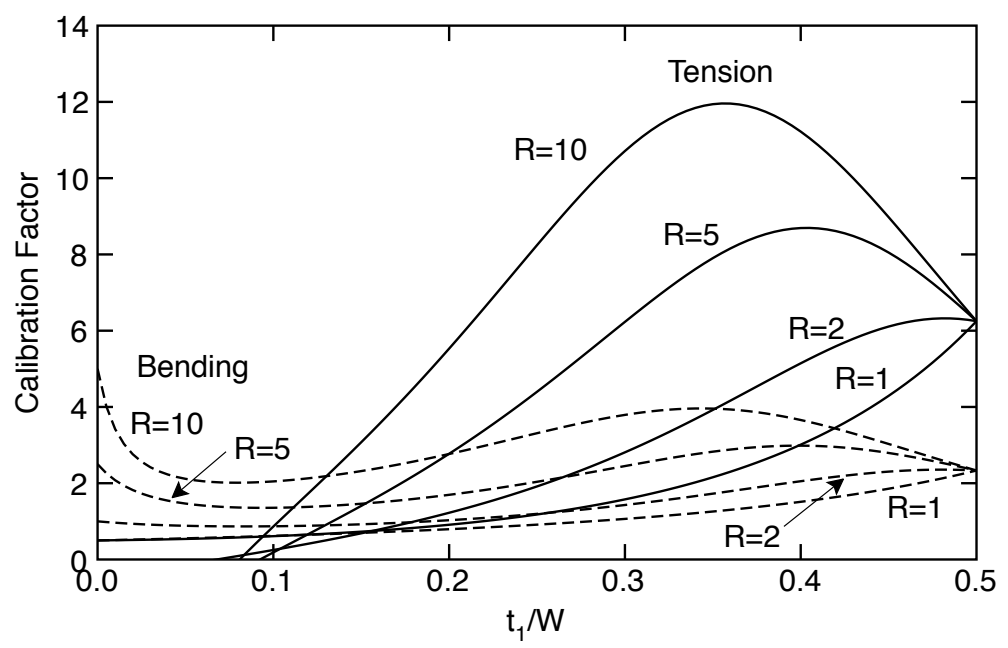

Fig. 13. Fracture mechanics calibration functions for longitudinal splitting of a coating off a core for mechanical loading only as a function of face thickness and for various stiffness ratios $R=E_{1} / E_{2}$. The solid curves are for tensile loading (see Eq. (70)). The dashed curves are for three-point bending (see Eq. (71)).

where $\kappa>0$ corresponds to curvature upward, $y=0$ is the midplane of the sublaminate, and positive $y$ is directed up. The axial stress in each layer, including residual stresses, is

$$
\sigma_{x x}(y)=E(y)\left(\varepsilon_{0}-\kappa y-\alpha(y) \Delta T\right)
$$

where $E(y)$ and $\alpha(y)$ are the position-dependent modulus and thermal expansion coefficient in the $x$ direction. Integrating these stresses, the total axial force, $N$, and bending moment, $M$, can be written as

$$
\begin{aligned}
N=\int_{A} \sigma_{x x}(y) d A & =-S_{n 1}^{(i)} \kappa+S_{n 2}^{(i)} \varepsilon_{0}-S_{n 3}^{(i)} \Delta T \\
M=-\int_{A} \sigma_{x x}(y) y d A & =S_{m 1}^{(i)} \kappa-S_{m 2}^{(i)} \varepsilon_{0}+S_{m 3}^{(i)} \Delta T
\end{aligned}
$$

where

$$
\begin{aligned}
S_{n 1}^{(i)}=S_{m 2}^{(i)} & =B \int_{-h / 2}^{h / 2} y E(y) d y=B \sum_{j=1}^{n_{i}} E_{j} t_{j} \overline{y_{j}} \\
S_{n 2}^{(i)} & =B \int_{-h / 2}^{h / 2} E(y) d y=B \sum_{j=1}^{n_{i}} E_{j} t_{j} \\
S_{m 1}^{(i)} & =B \int_{-h / 2}^{h / 2} y^{2} E(y) d y=B \sum_{j=1}^{n_{i}} E_{j} t_{j}\left(\bar{y}_{j}^{2}+\frac{t_{j}^{2}}{12}\right) \\
S_{n 3}^{(i)} & =B \int_{-h / 2}^{h / 2} E(y) \alpha(y) d y=B \sum_{j=1}^{n_{i}} E_{j} \alpha_{j} t_{j} \\
S_{m 3}^{(i)} & =B \int_{-h / 2}^{h / 2} y E(y) \alpha(y) d y=B \sum_{j=1}^{n_{i}} E_{j} \alpha_{j} t_{j} \bar{y}_{j}
\end{aligned}
$$

Here $E_{j}, \alpha_{j}, t_{j}$, and $\overline{y_{j}}$ are the $x$-direction modulus, $x$-direction thermal expansion coefficient, thickness, and midpoint of layer $j$ in sublaminate $i$ with $n_{i}$ layers. Inverting these equations leads to effective properties for sublaminate $i$ and equations for curvature and strain:

$$
\begin{aligned}
\kappa & =C_{\kappa}^{(i)} M+D^{(i)} N+\alpha_{\kappa}^{(i)} \Delta T \\
\varepsilon_{0} & =D^{(i)} M+C_{\varepsilon}^{(i)} N+\alpha_{\varepsilon}^{(i)} \Delta T
\end{aligned}
$$


where

$$
\begin{array}{rlrl}
C_{\kappa}^{(i)} & =\frac{S_{n 2}^{(i)}}{S_{m 1}^{(i)} S_{n 2}^{(i)}-S_{n 1}^{(i)} S_{m 2}^{(i)}} & C_{\varepsilon}^{(i)}=\frac{S_{m 1}^{(i)}}{S_{m 1}^{(i)} S_{n 2}^{(i)}-S_{n 1}^{(i)} S_{m 2}^{(i)}} & D^{(i)}=\frac{S_{m 2}^{(i)}}{S_{m 1}^{(i)} S_{n 2}^{(i)}-S_{n 1}^{(i)} S_{m 2}^{(i)}} \\
\alpha_{\kappa}^{(i)}=\frac{S_{m 2}^{(i)} S_{n 3}^{(i)}-S_{n 2}^{(i)} S_{m 3}^{(i)}}{S_{m 1}^{(i)} S_{n 2}^{(i)}-S_{n 1}^{(i)} S_{m 2}^{(i)}} & \alpha_{\varepsilon}^{(i)}=\frac{S_{m 1}^{(i)} S_{n 3}^{(i)}-S_{n 1}^{(i)} S_{m 3}^{(i)}}{S_{m 1}^{(i)} S_{n 2}^{(i)}-S_{n 1}^{(i)} S_{m 2}^{(i)}}
\end{array}
$$

The residual stresses in Eq. (10) are found from a beam analysis with $N=M=0$, which gives $\kappa=$ $\alpha_{\kappa}^{(i)} \Delta T, \varepsilon_{0}=\alpha_{\varepsilon}^{(i)} \Delta T$ and residual stress

$$
\sigma_{x x}^{r}(y)=E(y)\left(\alpha_{\varepsilon}^{(i)} \Delta T-\alpha_{\kappa}^{(i)} \Delta T y-\alpha(y) \Delta T\right)
$$

Subsitution into Eq. (10) for a small region of length $\Delta x=x_{1}-x_{0}$ gives

$$
\frac{1}{2} \int_{V} \boldsymbol{\sigma}^{r} \cdot \boldsymbol{\alpha} \Delta T d V=\Delta x \frac{\Delta T^{2}}{2}\left(S_{n 3}^{(i)} \alpha_{\varepsilon}^{(i)}-S_{m 3}^{(i)} \alpha_{\kappa}^{(i)}-S_{n 4}^{(i)}\right)
$$

where

$$
S_{n 4}^{(i)}=B \int_{-h / 2}^{h / 2} E(y) \alpha(y)^{2} d y=B \sum_{j=1}^{n_{i}} E_{j} \alpha_{j}^{2} t_{j}
$$

Using the relation

$$
\alpha_{\varepsilon}^{(i)}=\frac{S_{n 3}^{(i)}}{S_{n 2}^{(i)}}+\frac{S_{n 1}^{(i)}}{S_{n 2}^{(i)}} \alpha_{\kappa}^{(i)}
$$

leads to

$$
\frac{1}{2} \int_{V} \boldsymbol{\sigma}^{r} \cdot \boldsymbol{\alpha} \Delta T d V=\Delta x \frac{\Delta T^{2}}{2}\left(\frac{S_{n 3}^{(i)^{2}}}{S_{n 2}^{(i)}}-S_{n 4}^{(i)}+\frac{\alpha_{\kappa}^{(i)}{ }^{2}}{C_{\kappa}^{(i)}}\right)
$$

Substituting the definitions for $S_{n 2}^{(i)}, S_{n 3}^{(i)}$, and $S_{n 4}^{(i)}$, the result can be written as

$$
\frac{1}{2} \int_{V} \boldsymbol{\sigma}^{r} \cdot \boldsymbol{\alpha} \Delta T d V=\Delta x \frac{\Delta T^{2}}{2}\left(-B h E_{0}^{(i)}{\sigma_{\alpha E}^{(i)}}^{2}+\frac{\left.\alpha_{\kappa}^{(i)}\right)^{2}}{C_{\kappa}^{(i)}}\right)
$$

where $B h E_{0}^{(i)}=S_{n 2}^{(i)}$ and ${\sigma_{\alpha E}^{(i)}}^{2}=\left\langle\alpha^{2}\right\rangle_{E}-\langle\alpha\rangle_{E}^{2}$ is the variance of the modulus weighted thermal expansion coefficient with the averages defined by

$$
\left\langle\alpha^{2}\right\rangle_{E}=\frac{S_{n 4}^{(i)}}{S_{n 2}^{(i)}} \quad \text { and } \quad\langle\alpha\rangle_{E}=\frac{S_{n 3}^{(i)}}{S_{n 2}^{(i)}}
$$

For a layered sublaminate, the variance term can be written as

$$
B h E_{0}^{(i)}{\sigma_{\alpha E}^{(i)}{ }^{2}}_{\alpha}=\frac{B \sum_{j, k: j<k}^{n_{i}} E_{j} E_{k} t_{j} t_{k} \Delta \alpha_{j k}^{2}}{\sum_{j=1}^{n_{i}} E_{j} t_{j}}
$$

where $\Delta \alpha_{j k}=\alpha_{j}-\alpha_{k}$.

Several examples in this paper considered symmetric specimens with two materials having moduli $E_{1}$ and $E_{2}$ in the ratio $R=E_{1} / E_{2}$ with central layer having thickness $t_{2}$ and two outer layers having thickness $t_{1}$. When the crack runs at some location in the central layer, arms 1 and 2 will have two layers and will 
have thicknesses $h_{i}=t_{1}\left(1+\lambda_{i}\right) / \lambda_{i}$ where $\lambda_{i}$ is the ratio of $t_{1}$ to the amount of layer 2 in that arm. When layer 1 is on the top, the sums are:

$$
\begin{gathered}
S_{n 1}^{(i)}=S_{m 2}^{(i)}=\frac{B E_{1} t_{1}^{2}}{2 R \lambda_{i}}(R-1) \quad S_{n 2}^{(i)}=\frac{B E_{1} t_{1}}{R \lambda_{i}}\left(1+R \lambda_{i}\right) \quad S_{m 1}^{(i)}=\frac{B E_{1} t_{1}^{3}}{12 R \lambda_{i}^{3}}\left(1+3 R \lambda_{i}+3 \lambda_{i}^{2}+R \lambda_{i}^{3}\right) \\
S_{n 3}^{(i)}=\frac{B E_{1} t_{1}}{R \lambda_{i}}\left(R \lambda_{i} \alpha_{1}+\alpha_{2}\right) \quad S_{m 3}^{(i)}=\frac{B E_{1} t_{1}^{2}}{2 R \lambda_{i}}\left(R \alpha_{1}-\alpha_{2}\right)
\end{gathered}
$$

The thermomechanical properties for this two-layer beam are

$$
\begin{aligned}
C_{\kappa}^{(i)} & =\frac{12}{B E_{1} t_{1}^{3}} \frac{R \lambda_{i}^{3}\left(1+R \lambda_{i}\right)}{4 R \lambda_{i}\left(1+\lambda_{i}+\lambda_{i}^{2}\right)+\left(1+R \lambda_{i}^{2}\right)^{2}} \\
C_{\varepsilon}^{(i)} & =\frac{1}{B E_{1} t_{1}} \frac{R \lambda_{i}\left(1+3 R \lambda_{i}+3 \lambda_{i}^{2}+R \lambda_{i}^{3}\right)}{4 R \lambda_{i}\left(1+\lambda_{i}+\lambda_{i}^{2}\right)+\left(1+R \lambda_{i}^{2}\right)^{2}} \\
D^{(i)} & =\frac{6}{B E_{1} t_{1}^{2}} \frac{R \lambda_{i}^{3}(R-1)}{4 R \lambda_{i}\left(1+\lambda_{i}+\lambda_{i}^{2}\right)+\left(1+R \lambda_{i}^{2}\right)^{2}} \\
\alpha_{\kappa}^{(i)} & =-\frac{6 \Delta \alpha}{t_{1}} \frac{R \lambda_{i}^{2}\left(1+\lambda_{i}\right)}{4 R \lambda_{i}\left(1+\lambda_{i}+\lambda_{i}^{2}\right)+\left(1+R \lambda_{i}^{2}\right)^{2}} \\
\alpha_{\varepsilon}^{(i)} & =\frac{R \lambda_{i} \alpha_{1}\left(1+3 \lambda_{i}+3 \lambda_{i}^{2}+R \lambda_{i}^{3}\right)+\alpha_{2}\left(1+3 R \lambda_{i}+3 R \lambda_{i}^{2}+R \lambda_{i}^{3}\right)}{4 R \lambda_{i}\left(1+\lambda_{i}+\lambda_{i}^{2}\right)+\left(1+R \lambda_{i}^{2}\right)^{2}} \\
B h_{i} E_{0}^{(i)} \sigma_{\alpha E}^{(i)}{ }^{2} & =\frac{B E_{1} t_{1} \Delta \alpha^{2}}{1+R \lambda_{i}}
\end{aligned}
$$

where $\Delta \alpha=\alpha_{1}-\alpha_{2}$. When layer 2 is on top, $S_{n 1}^{(i)}, S_{m 2}^{(i)}$, and $S_{m 3}^{(1)}$ change sign leading to identical thermomechanical properties except for changes of sign in $D^{(i)}$ and $\alpha_{\kappa}^{(i)}$.

The intact part of the three-layer specimen specimen will be a symmetric three layer composite with the following non-zero sums

$$
S_{n 2}^{(3)}=\frac{B E_{1} t_{1}}{R \lambda}(1+2 R \lambda) \quad S_{m 1}^{(3)}=\frac{B E_{1} t_{1}^{3}}{12 R \lambda^{3}}\left(1+6 R \lambda+12 R \lambda^{2}+8 R \lambda^{3}\right) \quad S_{n 3}^{(3)}=\frac{B E_{1} t_{1}}{R \lambda}\left(2 R \lambda \alpha_{1}+\alpha_{2}\right)
$$

The non-zero thermomechanical properties for the symmetric, three-layer composite are

$$
\begin{gathered}
C_{\kappa}^{(3)}=\frac{12}{B E_{1} t_{1}^{3}} \frac{R \lambda^{3}}{1+6 R \lambda+12 R \lambda^{2}+8 R \lambda^{3}} \quad C_{\varepsilon}^{(3)}=\frac{1}{B E_{1} t_{1}} \frac{R \lambda}{1+2 R \lambda} \\
\alpha_{\varepsilon}^{(3)}=\frac{2 R \lambda \alpha_{1}+\alpha_{2}}{1+2 R \lambda} \quad B h_{3} E_{0}^{(3)} \sigma_{\alpha E}^{(3)^{2}}=\frac{2 B E_{1} t_{1} \Delta \alpha^{2}}{1+2 R \lambda}
\end{gathered}
$$

Acknowledgements. This work was supported by a grant from the Department of Energy DE-FG03-02ER45914 and by the University of Utah Center for the Simulation of Accidental Fires and Explosions (C-SAFE), funded by the Department of Energy, Lawrence Livermore National Laboratory, under Subcontract B341493.

\section{References}

1. Schapery, R. A. and B. D. Davidson, "Prediction of Energy Release rate for Mixed-Mode Delamination Using Classical Plate Theory," Appl. Mech. Rev., 43 (1990), S281-S287.

2. Hutchinson, J. W. and Z. Suo, "Mixed Mode Cracking in Layered Materials," Advances in Applied Mechanics, 29 (1992), 63-191.

3. Williams, J. G., "On the Calculation of Energy Release Rates for Cracked Laminates," Int. J. Fract., 36 (1988), 101-119. 
4. Nairn, J. A., "Energy Release Rate Analysis of Adhesive and Laminate Double Cantilever Beam Specimens Emphasizing the Effect of Residual Stresses," Int. J. Adhesion \& Adhesives, 20 (1999), 59-70.

5. Nairn, J. A., "Fracture Mechanics of Composites with Residual Thermal Stresses," J. Appl. Mech., 64 (1997), 804-810.

6. Nairn, J. A., "Exact and Variational Theorems for Fracture Mechanics of Composites with Residual Stresses, Traction-Loaded Cracks, and Imperfect Interfaces," Int. J. Fract., 105 (2000), 243-271.

7. Guo, S., D. A. Dillard, and J. A. Nairn, "The Effect of Residual Stress on the Energy Release Rate of Wedge and DCB Test Specimens," Int. J. Adhes. EJ Adhes., 26 (2006), 285-294.

8. Carlsson, L. A., J. W. Gillespire, and R. B. Pipes, "On The Analysis and Design of The End Notched Flexure (ENF) Specimen for Mode II Testing," J. Comp. Mater., 20 (1986), 594-604.

9. Nairn, J. A., "Finite Element and Material Point Method Software," http://oregonstate.edu/ nairnj/, 2006.

10. Sethuraman, R. and S. K. Maiti, "Finite Element Based Computation of Strain Energy Release Rate by Modified Crack Closure Integral," Eng. Fract. Mech., 30 (1988), 227-331.

11. Nairn, J. A., "Material Point Method Calculations with Explicit Cracks," Computer Modeling in Engineering 83 Sciences, 4 (2003), 649-664.

12. Guo, Y. and J. A. Nairn, "Calculation of J-Integral and Stress Intensity Factors using the Material Point Method," Computer Modeling in Engineering 63 Sciences, 6 (2004), 295-308.

13. Bardenhagen, S. G., J. E. Guilkey, K. M. Roessig, J. U. Brackbill, W. M. Witzel, and J. C. Foster, "An Improved Contact Algorithm for the Material Point Method and Application to Stress Propagation in Granular Material," Computer Modeling in Engineering 85 Sciences, 2 (2001), 509-522.

14. Hashemi, S., A. J. Kinloch, and J. G. Williams, "The Analysis of Interlaminar Fracture in Uniaxial Fibre Reinforced Polymer Composites," Proc. R. Soc. Lond., A347 (1990), 173-199.

15. Williams, J. G., "Fracture in Adhesive Joints: The Beam on Elastic Foundation Model," Proc. Int'l Mechanical Engineering Congress and Exhibition: The Winter Annual Meeting of the ASME, Symposium on Mechanics of Plastics and Plastic Composites, San Francisco, CA, USA, 12-17 November, 1995.

16. Nairn, J. A., "Energy Release Rate Analysis of Adhesive and Laminate Double Cantilever Beam Specimens Emphasizing the Effect of Residual Stresses," Int. J. Adhesion $\mathcal{E}$ Adhesives, 20 (1999), 59-70.

17. Davidson, B. D. and V. Sundararaman, "A Single-Leg Bending Test for Interfacial Fracture Toughness Determination," Int. J. Fract., 78 (1996), 193-210.

18. Pax, G. M., "Aminosilanes and Hyperbranched Polymers for Adhesion Tailoring Between Metallic Oxides and Polyethylene," Ph.D. Thesis, École Polytechnique Fédérale de Lausanne, Switzerland, 2005.

19. Cantwell, W. J., R. Scudamore, J. Ratcliffe, and P. Davies, "Interfacial Fracture in Sandwich Laminates," Comp. Sci. \& Tech., 59 (1999), 2079-2085.

20. W. Fawcett, "Comparison of Carbon Foam vs. Aluminum Honeycomb for Composite Cores at Elevated Temperatures," M.S. Thesis, University of Utah, Salt Lake City, UT, USA, 2005.

21. Nairn, J. A., "Fracture Mechanics of Unidirectional Composites Using the Shear-Lag Model I: Theory," J. Comp. Mat., 22 (1988), 561-588.

22. Nairn, J. A., "Fracture Mechanics of Unidirectional Composites Using the Shear-Lag Model II: Experiment," J. Comp. Mat., 22 (1988), 589-600.

23. Nairn, J. A., S. Liu, H. Chen, and A. R. Wedgewood, "Longitudinal Splitting in Epoxy and K-Polymer Composites: Shear-Lag Analysis Including the Effect of Fiber Bridging," J. Comp. Mat., 25 (1990), 1086-1107. 